\title{
Optimized Signal Distortion for PAPR Reduction of OFDM Signals with IFFT/FFT Complexity via ADMM Approaches
}

\author{
Yongchao Wang, Member, IEEE, Yanjiao Wang and Qingjiang Shi
}

\begin{abstract}
In this paper, we propose two low-complexity optimization methods to reduce peak-to-average power ratio (PAPR) values of orthogonal frequency division multiplexing (OFDM) signals via alternating direction method of multipliers (ADMM). First, we formulate a non-convex signal distortion optimization model based on minimizing data carrier distortion such that the constraints are placed on PAPR and the power of free carriers. Second, to obtain the model's approximate optimal solution efficiently, we design two low-complexity ADMM algorithms, named ADMM-Direct and ADMM-Relax respectively. Third, we show that, in ADMM-Direct/-Relax, all the optimization subproblems can be solved semi-analytically and the computational complexity in each iteration is roughly $\mathcal{O}\left(\ell N \log _{2} \ell N\right)$, where $\ell$ and $N$ are over-sampling factor and carrier number respectively. Moreover, we show that the resulting solution of ADMM-Direct is guaranteed to be some Karush-Kuhn-Tucker (KKT) point of the non-convex model when the iteration algorithm is convergent. For ADMM-Relax, we prove that it has theoretically guaranteed convergence and can approach arbitrarily close to some KKT point of the model if proper parameters are chosen. Simulation results demonstrate the effectiveness of the proposed approaches.
\end{abstract}

Index Terms-Orthogonal frequency division multiplexing (OFDM), peak-to-average power ratio (PAPR), free carrier power overhead (FCPO), signal distortion, alternating direction method of multipliers (ADMM).

\section{INTRODUCTION}

$\mathbf{O}$ RTHOGONAL frequency division multiplexing (OFDM) is an important multi-carrier modulation technique which has been used widely in modern wireless communication systems since it has high bandwidth efficiency and powerful ability to resist the effects of multi-path fading [1]. However, a major drawback of OFDM signals is their high peak-to-average power ratio (PAPR). Since the transmitter's power amplifiers (PA) are peak-power limited, the large PAPR lets the wireless communication engineers face a difficult dilemma between signal distortion and power efficiency [2]. The above dilemma can be seen from the following facts: On the one hand, to achieve high power amplifier efficiency, one can move working-point approaching to nonlinear region. Then, large signals would suffer from severe nonlinear distortion; On the other hand, to release nonlinear distortion of the large signals, one must move working-point back away from nonlinear region. Then, power efficiency would be low.

Over the past decades, there have been a variety of PAPR reduction techniques proposed in the literatures, which can be roughly classified into three categories: multiple signaling and probabilistic techniques, coding techniques, and signal distortion techniques [3]. The ideas of multiple signaling and probabilistic techniques, such as selective mapping (SLM) [4], partial transmit sequence (PTS) [5], tone reservation (TR) [6], constellation shaping [7], etc., are used to generate multiple permutations of the OFDM signals and transmit the one with a minimum PAPR, or to modify the OFDM signals by introducing phase shifts, adding peak reduction carriers, or changing constellation points to reduce the OFDM signals' PAPR. The coding techniques use some coding schemes, for example low density parity-check (LDPC) code [8], Hadamard code [9], etc., to perform PAPR reduction. Signal distortion techniques reduce the PAPR by distorting the transmitted OFDM signal before it passes through the PA. In comparison with other PAPR reduction techniques, signal distortion techniques have an important merit, which is that the signal distortion module can be inserted into the OFDM system directly and the corresponding transceiver's structure does not need to be changed. Repeated clipping and filtering (RCF) [10] may be the simplest signal distortion method in the sense of computational complexity, which in every iteration is dominant by one fast fourier transform (FFT) operation and one inverse FFT (IFFT) operation. However, on the one hand, the classical RCF technique and its variants, such as companding transform [11], peak windowing [12], peak cancellation [13], etc., cannot meet more complicated practical demands, such as controlling free carrier power under the specified level or achieving optimized signal distortion while approaching the desired PAPR values. On the other hand, as iteration algorithms, complete convergence analysis of these methods is still unavailable.

In recent years, signal distortion techniques based on optimization methods have been exploited to reduce the PAPR of OFDM signals while achieving optimal system parameters. These kinds of optimization methods can make up the performance of the existing signal distortion methods, such as PAPR values and the corresponding signal distortion. Second order conic programming (SOCP) approach was one of the widely used techniques, which is exploited to minimize peaks of the time-domain waveforms subject to constraints on error vector magnitude (EVM) and the free carrier power overhead (FCPO) [14]. After that, several SOCP approaches were proposed to improve the PAPR performance of OFDM signals [15]-[18]. Semi-definite programming (SDP) is another important optimization technique to reduce the PAPR of the OFDM signals. In [19], the authors exploited the semi- 
definite relaxation technique to relax the non-convex quadratic optimization model for OFDM signals and showed that the optimized OFDM symbols have a quasi-constant PAPR value.

The main concern of the existing PAPR optimization methods is their high-computational complexity. In this paper, we focus on this issue and develop two low-complexity optimization methods via the alternating direction method of multipliers (ADMM) technique, whose complexities are comparable to the classical RCF method and also are calculated as dominant by one FFT operation and one IFFT operation. The main content of this paper is as follows: first, we establish a nonconvex signal distortion optimization model which is based on minimizing data carrier distortion such that the constraints are placed on PAPR and the power of the free carriers. To obtain approximate optimal solution of the non-convex model efficiently, we exploit the ADMM technique [20]- [23], and propose two customized ADMM algorithms, named ADMMDirect and ADMM-Relax respectively. It can be shown that, in both of the proposed algorithms, all the subproblems' optimal solutions can be determined semi-analytically and the computational complexity in each iteration is roughly $\mathcal{O}\left(\ell N \log _{2} \ell N\right)$, where $\ell$ and $N$ are the over-sampling factor and carrier number respectively. Moreover, we show that the resulting solution of the ADMM-Direct algorithm is guaranteed to be some Karush-Kuhn-Tucker (KKT) point of the considered model when the iteration algorithm is convergent. For ADMM-Relax, we prove that it is convergent and can approach arbitrarily close to some KKT point of the model if proper parameters are chosen. Furthermore, the proposed ADMM algorithms outperform the existing approaches. For example, not only the desired OFDM symbols with quasiconstant PAPR values and small signal distortion can be obtained just after a few iterations but also convergence is theoretically guaranteed.

The rest of this paper is organized as follows. Section II introduces preliminaries related to OFDM signals and the considered OFDM optimization model. In Section III and IV, we exploit the ADMM technique and propose two lowcomplexity algorithms, named ADMM-Direct and ADMMRelax respectively. Their performance analysis, such as convergence, convergence rate and complexity, are presented. Simulation results are shown to evaluate the performance of the proposed low-complexity OFDM PAPR reduction algorithms in Section V. Section VI concludes this paper.

Notations: In this paper, bold lowercase and uppercase letters denote vectors and matrices respectively; $(\cdot)^{T}$ and $(\cdot)^{H}$ symbolize the transpose and conjugate transpose operations; 2 -norm of a vector $\mathbf{a}, \infty$-norm of a vector $\mathbf{a}$ and the Frobenius norm of a matrix $\mathbf{A}$ are denoted by $\|\mathbf{a}\|_{2},\|\mathbf{a}\|_{\infty}$ and $\|\mathbf{A}\|_{\mathrm{F}}$ respectively; $\nabla$ denotes the gradient operator. $\dagger$ denotes pseudo-inverse operator.

\section{PRELIMINARIES}

Consider an OFDM system with $N$ carriers. Let $\mathbf{c} \in \mathbb{C}^{N}$ denote an OFDM frequency-domain symbol and $\mathrm{x} \in \mathbb{C}^{\ell N}$ be its corresponding time-domain symbol. Let $\mathbf{A} \in \mathbb{C}^{\ell N \times N}$ be the first $N$ columns of the $\ell N$-points IDFT matrix. Then, there are

$$
\begin{aligned}
& \mathbf{x}=\mathbf{A} \mathbf{c}=\operatorname{IFFT}_{\ell}(\mathbf{c}) \sqrt{1}, \\
& \mathbf{c}=\ell N \mathbf{A}^{H} \mathbf{x}=\operatorname{FFT}_{\ell}(\mathbf{x}),
\end{aligned}
$$

where $\ell$ is the over-sampling factor, $\operatorname{IFFT}_{\ell}(\mathbf{c})$ denotes $\ell N$ points IFFT operation for the frequency-domain symbol $\mathbf{c}$ with $\ell$-times over-sampling, and $\mathrm{FFT}_{\ell}(\mathbf{x})$ denotes $\ell N$-points FFT operation for the time-domain symbol $\mathbf{x}$, but only outputs the first $N$ elements.

PAPR of the time-domain OFDM symbol $\mathbf{x}$ is defined as

$$
\text { PAPR : } \frac{\max _{i=1, \ldots, \ell N}\left|x_{i}\right|^{2}}{\frac{1}{\ell N} \sum_{i=1}^{\ell N}\left|x_{i}\right|^{2}}=\frac{\|\mathbf{x}\|_{\infty}^{2}}{\frac{1}{\ell N}\|\mathbf{x}\|_{2}^{2}} .
$$

From (1) and (2), we see that there could exist large peaks in the time-domain OFDM symbol if carriers in the frequencydomain OFDM symbol are in phase or nearly in phase.

In many OFDM systems, carriers in an OFDM symbol consist of data carriers and free carriers. The former are exploited to carry information and the latter are reserved to control the out-of-band emission or some future possible applications. Generally, both introducing small distortion in the data carriers and assigning some controlled power to the free carriers can change PAPR values of the OFDM symbols. Based on these observations, we combine PAPR, data carrier distortion, free carrier power together and formulate the following optimization model

$$
\begin{array}{ll}
\min _{\mathbf{c} \in \mathbb{C}^{N}, \mathbf{x} \in \mathbb{C}^{\ell N}} & \frac{1}{2}\left\|\mathbf{S}_{\mathrm{D}}\left(\mathbf{c}-\mathbf{c}_{\mathrm{o}}\right)\right\|_{2}^{2}, \\
\text { subject to } & \frac{\|\mathbf{x}\|_{\infty}^{2}}{\frac{1}{\ell N}\|\mathbf{x}\|_{2}^{2}}=\alpha \\
& \frac{\left\|\mathbf{S}_{\mathrm{F}} \mathbf{c}\right\|_{2}^{2}}{\left\|\mathbf{S}_{\mathrm{D}} \mathbf{c}\right\|_{2}^{2}} \leq \beta \\
& \mathbf{A c}=\mathbf{x} .
\end{array}
$$

In the model (3), $\mathbf{c}_{\mathrm{o}}$ is the original OFDM symbol. The matrix $\mathbf{S}_{\mathrm{D}}$ is binary and diagonal. The corresponding set $\mathrm{D}=\left\{i_{m} \mid m=1, \cdots, M\right\}$ and $i_{m}$ labels the $m$ th data carrier. $\mathbf{S}_{\mathrm{D} i i}=1$ if $i \in \mathrm{D}$ and $\mathbf{S}_{\mathrm{D} i i}=0$ otherwise. The matrix $\mathbf{S}_{\mathrm{F}}$ and index set $\mathrm{F}$ have similar definitions except for the free carriers. The constraints $3 \mathrm{~b}$ and $3 \mathrm{c}$ are PAPR constraint and free carriers constraint respectively, where $\alpha$ and $\beta$ are pre-set thresholds.

We have the following comments on the model (3):

- Direct minimizing peak values of the OFDM time domain symbols is another optimization strategy. In comparison with it, the main benefit of the model (3) is that the optimized OFDM symbols have almost quasi-constant PAPR values, which can help us choose a proper working point of nonlinear PA.

- According to the definitions of the matrices $\mathbf{S}_{\mathrm{D}}$ and $\mathbf{S}_{\mathrm{F}}$, it can be seen that $\mathbf{S}_{\mathrm{D}}+\mathbf{S}_{\mathrm{F}}=\mathbf{I}$ and $\mathbf{S}_{\mathrm{D}} \mathbf{S}_{\mathrm{F}}=\mathbf{0}$, where

\footnotetext{
${ }^{1} \mathbf{x}$ can be cast as some discrete signal sampled from continuous time domain OFDM signal.
} 
I is an identity matrix.

- To guarantee that the feasible region of the model (3) is non-empty, the pre-set thresholds $\alpha$ and $\beta$ should be set no less than 1 and 0 respectively, i.e., $\alpha \geq 1$ and $\beta \geq 0$.

- The model (3) is designed to optimize the OFDM symbol whose PAPR is larger than $\alpha$. So, if the considered OFDM symbol's PAPR is less than $\alpha$, we do not process it and pass it to PA directly.

- Since the constraints (3b) and (3c) are non-convex, it is difficult to obtain its global optimizer of the model (3). Existing techniques, such as semi-definite relaxation, can be exploited to relax the model (3) to be convex and generate approximate optimal solutions. However, its computational cost is roughly $\mathcal{O}\left(\ell^{3.5} N^{3.5}\right)$, which is prohibitive in practice. In the sequel, two low-complexity algorithms based on the ADMM technique for the model (3) are proposed and we show that their computational complexities in each iteration are roughly $\mathcal{O}\left(\ell N \log _{2} \ell N\right)$. Moreover, the desired OFDM symbol with quasi-constant PAPR values and optimized signal distortion can be obtained just after a few iterations and the proposed iteration algorithms have theoretically guaranteed convergence. In comparison with the proposed ADMM algorithms, the RCF method does not have these kinds of theoretical results.

\section{Solving ALGORIthm I: ADMM-DiReCT}

ADMM is a simple but powerful technique that solves large scale optimization problems by breaking them into small ones, each of which is then easier to handle. In this section, we propose the ADMM-Direct algorithm, which solves the problem (3) via the ADMM technique directly. In ADMMDirect, all the subproblems' optimal solutions can be determined semi-analytically and the computational complexity in each iteration is roughly $\mathcal{O}\left(\ell N \log _{2} \ell N\right)$. Moreover, we prove that the resulting solution of the ADMM-Direct algorithm is guaranteed to be some KKT point of the model (3) when the algorithm is convergent.

\section{A. ADMM-Direct Algorithm Framework}

The proposed ADMM-Direct algorithm is shown as follows.

$$
\begin{aligned}
& \mathbf{c}^{k+1}=\underset{\mathbf{c} \in \mathcal{C}}{\arg \min } L_{\rho}\left(\mathbf{c}, \mathbf{x}^{k}, \mathbf{y}^{k}\right), \\
& \mathbf{x}^{k+1}=\underset{\mathbf{x} \in \mathcal{X}}{\arg \min } L_{\rho}\left(\mathbf{c}^{k+1}, \mathbf{x}, \mathbf{y}^{k}\right), \\
& \mathbf{y}^{k+1}=\mathbf{y}^{k}+\rho\left(\mathbf{A c}^{k+1}-\mathbf{x}^{k+1}\right) .
\end{aligned}
$$

In (4), $L_{\rho}(\mathbf{c}, \mathbf{x}, \mathbf{y})$ is the augmented Lagrangian function of the model (3) and it can be expressed as 2

$$
\begin{aligned}
L_{\rho}(\mathbf{c}, \mathbf{x}, \mathbf{y})= & \frac{1}{2}\left\|\mathbf{S}_{\mathrm{D}}\left(\mathbf{c}-\mathbf{c}_{\mathrm{o}}\right)\right\|_{2}^{2}+\operatorname{Re}\left(\mathbf{y}^{H}(\mathbf{A} \mathbf{c}-\mathbf{x})\right) \\
& +\frac{\rho}{2}\|\mathbf{A} \mathbf{c}-\mathbf{x}\|_{2}^{2},
\end{aligned}
$$

where $\rho>0$ is the penalty parameter, $\mathbf{x} \in \mathcal{X}$ and $\mathbf{c} \in \mathcal{C}$ denote the constraints (3b) and (3c) respectively, $\mathbf{y} \in \mathbb{C}^{\ell N}$ is the Lagrangian multiplier, and $k$ is the iteration number.

The challenges of implementing ADMM-Direct (4) are how to solve (4ab) and (4b) since their corresponding constraints are non-convex. In the following, we show that both of them can be obtained effectively by exploiting the structure of (3).

\section{B. Solving the Subproblem (4a}

Based on the augmented Lagrangian function $L_{\rho}(\mathbf{c}, \mathbf{x}, \mathbf{y})$, the problem (4a) can be equivalent to

$$
\begin{aligned}
& \underset{\mathbf{c} \in \mathbb{C}^{N}}{\min _{\text {subject to }}} \frac{1}{2}\left\|\mathbf{S}_{\mathrm{D}}\left(\mathbf{c}-\mathbf{c}_{\mathrm{o}}\right)\right\|_{2}^{2}+\frac{\rho}{2}\|\mathbf{c}\|_{2}^{2}-\beta\left\|\mathbf{S}_{\mathrm{D}} \mathbf{c}\right\|_{2}^{2} \leq 0 . \mathbf{x}^{k}+\frac{\mathbf{y}^{k}}{\rho} \|_{2}^{2},
\end{aligned}
$$

Since there is only one constraint in (6), its optimal solution can be determined through the Lagrangian multiplier method. The Lagrangian function of model (6) can be written as

$$
\begin{aligned}
L\left(\mathbf{c}, \mu^{k}\right)= & \frac{1}{2}\left\|\mathbf{S}_{\mathrm{D}}\left(\mathbf{c}-\mathbf{c}_{\mathrm{o}}\right)\right\|_{2}^{2}+\frac{\rho}{2}\left\|\mathbf{A} \mathbf{c}-\mathbf{x}^{k}+\frac{\mathbf{y}^{k}}{\rho}\right\|_{2}^{2} \\
& +\mu^{k}\left(\left\|\mathbf{S}_{\mathrm{F}} \mathbf{c}\right\|_{2}^{2}-\beta\left\|\mathbf{S}_{\mathrm{D}} \mathbf{c}\right\|_{2}^{2}\right),
\end{aligned}
$$

where the Lagrangian multiplier is $\mu^{k} \geq 0$. Since the problem (66) is feasible, the Lagrangian multiplier theorem indicates that its global optimal solution $\mathbf{c}^{k+1}$, combining the optimal Lagrangian multiplier $\mu^{k *}$, should satisfy $\nabla_{\mathbf{c}} L\left(\mathbf{c}^{k+1}, \mu^{k *}\right)=$ 0, i.e.,

$$
\begin{aligned}
\nabla_{\mathbf{c}} L\left(\mathbf{c}^{k+1}, \mu^{k *}\right)= & \mathbf{S}_{\mathrm{D}}\left(\mathbf{c}^{k+1}-\mathbf{c}_{\mathrm{o}}\right)+\rho \mathbf{A}^{H}\left(\mathbf{A} \mathbf{c}^{k+1}-\mathbf{x}^{k}+\frac{\mathbf{y}^{k}}{\rho}\right) \\
& +2 \mu^{k *}\left(\mathbf{S}_{\mathrm{F}}-\beta \mathbf{S}_{\mathrm{D}}\right) \mathbf{c}^{k+1}=0 .
\end{aligned}
$$

Since $\mathbf{A}^{H} \mathbf{A}=\frac{\mathbf{I}}{\ell N}$ and $\mathbf{c}_{\mathrm{o}}=\mathbf{S}_{\mathrm{D}} \mathbf{c}_{\mathrm{o}}$, we can further derive 8 as

$\left(\mathbf{S}_{\mathrm{D}}+\frac{\rho}{\ell N} \mathbf{I}+2 \mu^{k *}\left(\mathbf{S}_{\mathrm{F}}-\beta \mathbf{S}_{\mathrm{D}}\right)\right) \mathbf{c}^{k+1}=\mathbf{c}_{\mathrm{o}}+\rho \mathbf{A}^{H}\left(\mathbf{x}^{k}-\frac{\mathbf{y}^{k}}{\rho}\right)$.

Then, we can obtain

$$
\mathbf{c}^{k+1}=\left(\mathbf{S}_{\mathrm{D}}+\frac{\rho}{\ell N} \mathbf{I}+2 \mu^{k *}\left(\mathbf{S}_{\mathrm{F}}-\beta \mathbf{S}_{\mathrm{D}}\right)\right)^{\dagger} \mathbf{v}^{k},
$$

where $\mathbf{v}^{k}=\mathbf{c}_{\mathrm{o}}+\rho \mathbf{A}^{H}\left(\mathbf{x}^{k}-\frac{\mathbf{y}^{k}}{\rho}\right)$.

Now we consider how to determine $\mu^{k *}$. If the constraint (6b) is inactive, the corresponding Lagrangian multiplier $\mu^{k *}=0$. Otherwise, if the constraint (6b) is active, $\mathbf{c}^{k+1}$ should satisfy the constraint (6b) when " $="$ holds. We first consider the latter. Plugging (9) into $\left\|\mathbf{S}_{\mathrm{F}} \mathbf{c}^{k+1}\right\|_{2}$ and $\left\|\mathbf{S}_{\mathrm{D}} \mathbf{c}^{k+1}\right\|_{2}$, we have

$$
\begin{aligned}
\left\|\mathbf{S}_{\mathrm{F}} \mathbf{c}^{k+1}\right\|_{2} & =\frac{\left\|\mathbf{S}_{\mathrm{F}} \mathbf{v}^{k}\right\|_{2}}{\frac{\rho}{\ell N}+2 \mu^{k *}}, \\
\left\|\mathbf{S}_{\mathrm{D}} \mathbf{c}^{k+1}\right\|_{2} & =\frac{\left\|\mathbf{S}_{\mathrm{D}} \mathbf{v}^{k}\right\|_{2}}{1+\frac{\rho}{\ell N}-2 \mu^{k *} \beta} .
\end{aligned}
$$

${ }^{2}$ When the constraint is complex, one can introduce real Lagrangian multipliers $\mathbf{y}_{\mathrm{R}}$ and $\mathbf{y}_{\mathrm{I}}$ respectively for its real part and imaginary part. Then, according to the classical Lagrangian multiplier theory, the augmented Lagrangian function [5 can be derived easily, where $\mathbf{y}=\mathbf{y}_{\mathrm{R}}+j \mathbf{y}_{\mathrm{I}}$. 
Notice here we use the properties that $\mathbf{S}_{\mathrm{D}}$ and $\mathbf{S}_{\mathrm{F}}$ are diagonal matrices and $\mathbf{S}_{\mathrm{D}} \mathbf{S}_{\mathrm{F}}=0$. Plugging 10a and 10b into $\left\|\mathbf{S}_{\mathrm{F}} \mathbf{c}^{k+1}\right\|_{2}^{2}=\beta\left\|\mathbf{S}_{\mathrm{D}} \mathbf{c}^{k+1}\right\|_{2}^{2}$, we obtain

$$
\mu^{k *}=\frac{\left(1+\frac{\rho}{\ell N}\right)\left\|\mathbf{S}_{\mathrm{F}} \mathbf{v}^{k}\right\|_{2}-\sqrt{\beta} \frac{\rho}{\ell N}\left\|\mathbf{S}_{\mathrm{D}} \mathbf{v}^{k}\right\|_{2}}{2\left(\beta\left\|\mathbf{S}_{\mathrm{F}} \mathbf{v}^{k}\right\|_{2}+\sqrt{\beta}\left\|\mathbf{S}_{\mathrm{D}} \mathbf{v}^{k}\right\|_{2}\right)} .
$$

In the implementation, we still need to know in what case we use (11) to compute $\mu^{k *}$ or just set $\mu^{k *}$ as zero. Observing (11), we see that the computed result for $\mu^{k *}$ could be negative. However, the Lagrangian multiplier theory guarantees that $\mu^{k *}$ should always be nonnegative since it is for the inequality constraint $6 \mathrm{~b}$ ). This contradiction comes from the assumption that the constraint is active. It means that the constraint is inactive and so $\mu^{k *}$ should be zero. Based on this observation, we can compute $\mu^{k *}$ by

$$
\mu^{k *}=\max \left\{0, \frac{\left(1+\frac{\rho}{\ell N}\right)\left\|\mathbf{S}_{\mathrm{F}} \mathbf{v}^{k}\right\|_{2}-\sqrt{\beta} \frac{\rho}{\ell N}\left\|\mathbf{S}_{\mathrm{D}} \mathbf{v}^{k}\right\|_{2}}{2\left(\beta\left\|\mathbf{S}_{\mathrm{F}} \mathbf{v}^{k}\right\|_{2}+\sqrt{\beta}\left\|\mathbf{S}_{\mathrm{D}} \mathbf{v}^{k}\right\|_{2}\right)}\right\} .
$$

TABLE I: Binary section searching procedure for $\gamma^{k *}$

Initialization: Set search boundary $\left(\gamma_{\text {left }}^{k}, \gamma_{\text {right }}^{k}\right)$. To guarantee $\gamma^{k *} \in\left(\gamma_{\text {left }}^{k}, \gamma_{\text {right }}^{k}\right)$, we set $\gamma_{\text {left }}^{k}=0$ and $\gamma_{\text {right }}^{k}$ is large enough.

Repeat: Let $\gamma^{k}=\frac{\gamma_{\text {left }}^{k}+\gamma_{\text {right }}^{k}}{2}$. Update $\mathbf{z}^{k+1}$ using (19). If $\left\|\mathbf{z}^{k+1}\right\|_{2}^{2}<1$, set $\gamma_{\text {right }}^{k}=\gamma^{k}$. Otherwise set $\gamma_{\text {left }}^{k}=\gamma^{k}$. Until $\left\|\mathbf{z}^{k+1}\right\|_{2}^{2}$ is close to 1 enough and let $\gamma^{k *}=\frac{\gamma_{\text {left }}^{k}+\gamma_{\text {right }}^{k}}{2}$.

\section{Solving the Subproblem (4b)}

Based on the augmented Lagrangian function $L_{\rho}(\mathbf{c}, \mathbf{x}, \mathbf{y})$, the subproblem (4b) can be equivalent to

$$
\begin{array}{ll}
\min _{\mathbf{x} \in \mathbb{C}^{\ell N}} & \left\|\mathbf{A c}^{k+1}-\mathbf{x}+\frac{\mathbf{y}^{k}}{\rho}\right\|_{2}^{2}, \\
\text { subject to } & \frac{\|\mathbf{x}\|_{\infty}^{2}}{\frac{1}{\ell N}\|\mathbf{x}\|_{2}^{2}}=\alpha .
\end{array}
$$

To simplify the constraint $13 \mathrm{~b}$, we introduce auxiliary variables $t$ and $\mathbf{z}$ to express $\mathbf{x}$ by $\mathbf{x}=t \mathbf{z}$, where $t>0$ and $\|\mathbf{z}\|_{2}^{2}=1$. Plugging them into [13, it is equivalent to

$$
\begin{aligned}
\min _{\mathbf{z} \in \mathbb{C}^{\ell N}, t>0} & t^{2}-2 t \operatorname{Re}\left(\mathbf{z}^{H} \mathbf{b}^{k}\right), \\
\text { subject to } & \left|z_{i}\right|^{2} \leq \frac{\alpha}{\ell N}, \quad i=1, \cdots, \ell N, \\
& \|\mathbf{z}\|_{2}^{2}=1,
\end{aligned}
$$

where $\mathbf{b}^{k}=\mathbf{A} \mathbf{c}^{k+1}+\frac{\mathbf{y}^{k}}{\rho}$ and $z_{i} \in \mathbf{z}$.

To be clear, we use $\mathbf{z}^{k+1}$ and $t^{k+1}$ to denote the optimal solutions of the model (14). Apparently, to minimize the objective 14a, $\operatorname{Re}\left(\mathbf{z}^{H} \mathbf{b}^{k}\right)$ should be maximized subject to (14b) and (14c). Specifically, we drop $t$ from the model (14) and formulate (15) to solve $\mathbf{z}^{k+1}$.

$$
\begin{array}{ll}
\max _{\mathbf{z} \in \mathbb{C}^{\ell N}} & \operatorname{Re}\left(\mathbf{z}^{H} \mathbf{b}^{k}\right), \\
\text { subject to } & \left|z_{i}\right|^{2} \leq \frac{\alpha}{\ell N}, \quad i=1, \cdots, \ell N, \\
& \|\mathbf{z}\|_{2}^{2}=1 .
\end{array}
$$

Moreover, we can further change $15 \mathrm{c}$ to an inequality constraint and formulate an equivalent convex optimization model (16), which can be solved as an SOCP problem with computational complexity $\mathcal{O}\left(\ell^{3} N^{3}\right)$ using free optimization solver [24] [25]. Here, we say that the models (15) and (16) are equivalent in the sense that both of them have the same optimal solution. We prove this fact in Appendix A.

$$
\begin{array}{ll}
\max _{\mathbf{z} \in \mathbb{C}^{\ell N}} & \operatorname{Re}\left(\mathbf{z}^{H} \mathbf{b}^{k}\right), \\
\text { subject to } & \left|z_{i}\right|^{2} \leq \frac{\alpha}{\ell N}, \quad i=1, \cdots, \ell N, \\
& \|\mathbf{z}\|_{2}^{2} \leq 1 .
\end{array}
$$

From a practical viewpoint, solving the problem (16) with complexity $\mathcal{O}\left(\ell^{3} N^{3}\right)$ is still expensive. In the following, we devise an inexact parallel solving algorithm, which can be implemented very effectively. First, we introduce the Lagrangian multiplier $\gamma^{k}>0$ for the constraint (16c) and rewrite (16) as

$$
\begin{array}{ll}
\min _{z_{i} \in \mathbb{C}, \gamma^{k}>0} & \sum_{i=1}^{\ell N}-\operatorname{Re}\left(z_{i}^{\dagger} b_{i}^{k}\right)+\gamma^{k}\left(\sum_{i=1}^{\ell N}\left|z_{i}\right|^{2}-1\right), \\
\text { subject to } & \left|z_{i}\right| \leq \sqrt{\frac{\alpha}{\ell N}}, \quad i=1, \cdots, \ell N,
\end{array}
$$

where " $\nmid$ " denotes the conjugate operator. Since both the objective function (17a) and constraint (17b) can be treated separately in $z_{i}$, solving the model 17 is equivalent to solving the following $\ell N$ subproblems (18), which can be implemented in parallel.

$$
\begin{array}{ll}
\min _{z_{i}} \in \mathbb{C}, \gamma^{k}>0 & -\operatorname{Re}\left(z_{i}^{\dagger} b_{i}^{k}\right)+\gamma^{k}\left|z_{i}\right|^{2}, \\
\text { subject to } & \left|z_{i}\right| \leq \sqrt{\frac{\alpha}{\ell N}} .
\end{array}
$$

Since 18a is a convex quadratic function and the constraint (18b) involves only one variable, the model's optimizer can be obtained by setting the objective's gradient as zero and then projecting the corresponding equation's solution onto the feasible region, i.e.,

$$
z_{i}^{k+1}=\left\{\begin{array}{cl}
\frac{b_{i}^{k}}{2 \gamma^{k}}, & \frac{\left|b_{i}^{k}\right|}{2 \gamma^{k}}<\sqrt{\frac{\alpha}{\ell N}} \\
\sqrt{\frac{\alpha}{\ell N}} j \phi\left(b_{i}^{k}\right), & \text { otherwise }
\end{array}\right.
$$

where $\phi\left(b_{i}^{k}\right)$ represents the phase of $b_{i}^{k}$. Moreover, the optimal Lagrangian multiplier $\gamma^{k *}$ can be obtained effectively through the binary section searching procedure as shown in Table [I. Then, plugging $\mathbf{z}^{k+1}$ into the model (14) and simplifying it as a quadratic problem, we can get

$$
t^{k+1}=\operatorname{Re}\left(\mathbf{z}^{k+1 H} \mathbf{b}^{k}\right) .
$$


According to (19), we can find that $\operatorname{Re}\left(\mathbf{z}^{k+1 H} \mathbf{b}^{k}\right)$ is guaranteed to be positive. Thus, the constraint $t>0$ is always satisfied. Plugging $\mathbf{z}^{k+1}$ and $t^{k+1}$ into $\mathbf{x}=t \mathbf{z}$, we get $\mathbf{x}^{k+1}$.

Initialization: Initialize $\left(\mathbf{c}^{1}, \mathbf{x}^{1}, \mathbf{y}^{1}\right)$. Choose parameters $(\alpha, \beta, \rho)$. Based on the considered OFDM scheme, set diagonal matrices $\mathbf{S}_{\mathrm{D}}$ and $\mathbf{S}_{\mathrm{F}}$.

For $k=1,2,3 \cdots$

S.1 Solve the subproblem (4a).

1.1 Compute $\mathbf{v}^{k}=\mathbf{c}_{\mathrm{o}}+\frac{\rho}{\ell N} \mathrm{FFT}_{\ell}\left(\mathbf{x}^{k}-\frac{\mathbf{y}^{k}}{\rho}\right)$.

1.2 Compute

$\mu^{k *}=\max \left\{0, \frac{\left(1+\frac{\rho}{\ell N}\right)\left\|\mathbf{S}_{\mathrm{F}} \mathbf{v}^{k}\right\|_{2}-\sqrt{\beta} \frac{\rho}{\ell N}\left\|\mathbf{S}_{\mathrm{D}} \mathbf{v}^{k}\right\|_{2}}{2\left(\beta\left\|\mathbf{S}_{\mathrm{F}} \mathbf{v}^{k}\right\|_{2}+\sqrt{\beta}\left\|\mathbf{S}_{\mathrm{D}} \mathbf{v}^{k}\right\|_{2}\right)}\right\}$.

1.3 Compute

$\mathbf{c}^{k+1}=\left(\mathbf{S}_{\mathrm{D}}+\frac{\rho}{\ell N} \mathbf{I}+2 \mu^{k *}\left(\mathbf{S}_{\mathrm{F}}-\beta \mathbf{S}_{\mathrm{D}}\right)\right)^{\dagger} \mathbf{v}^{k}$.

S.2 Solve the subproblem 4b.

2.1 Compute $\mathbf{b}^{k}=\operatorname{IFFT}_{\ell}\left(\mathbf{c}^{k+1}\right)+\frac{\mathbf{y}^{k}}{\rho}$.

2.2 Compute $\mathbf{z}^{k+1}$ through the binary section searching procedure in Table [

2.3 Compute $t^{k+1}=\max \left\{0, \operatorname{Re}\left(\mathbf{z}^{k+1 H} \mathbf{b}^{k}\right)\right\}$.

2.4 Compute $\mathbf{x}^{k+1}=t^{k+1} \mathbf{z}^{k+1}$.

S.3 Update Lagrangian multipliers.

Compute $\mathbf{y}^{k+1}=\mathbf{y}^{k}+\rho\left(\operatorname{IFFT}_{\ell}\left(\mathbf{c}^{k+1}\right)-\mathbf{x}^{k+1}\right)$.

Until some preset termination conditions are satisfied. Then, let $\mathrm{x}^{k+1}$ be the output.

Fig. 1: ADMM-Direct algorithm for the model (3).

\section{Performance Analysis on the ADMM-Direct Algorithm}

1) Computational complexity: In each ADMM-Direct iteration, the computational cost is quite cheap, which is comparable to the classical RCF approach [10]. For ADMMDirect algorithm scheme in Figure 1, we first consider the computational complexity of solving $\mathbf{c}^{k+1}$. In S.1.1, when we compute $\mathbf{v}^{k}$, it is obvious that the main cost lies in computing $\mathrm{FFT}_{\ell}\left(\mathbf{x}^{k}-\frac{\mathbf{y}^{k}}{\rho}\right)$. Since $\mathbf{x}^{k}-\frac{\mathbf{y}^{k}}{\rho}$ is an $\ell N$-length vector, the computational complexity to determine $\mathbf{v}^{k}$ is roughly $\mathcal{O}\left(\ell N \log _{2} \ell N\right)$. Notice here that $\ell N$ points FFT operation can be implemented through $\frac{1}{2} \ell N \log _{2} \ell N$ complex multiplications. In S.1.2, since $\mathrm{S}_{\mathrm{D}}$ and $\mathbf{S}_{\mathrm{F}}$ are binary and diagonal matrices, it costs only $\mathcal{O}(N)$ complex multiplications to obtain $\left\|\mathbf{S}_{\mathrm{D}} \mathbf{v}^{k}\right\|_{2}$ and $\left\|\mathbf{S}_{\mathrm{F}} \mathbf{v}^{k}\right\|_{2}$, i.e., the computational cost to determine $\mu^{k *}$ is roughly $\mathcal{O}(2 N)$. In S.1.3, since the matrix $\mathbf{S}_{\mathrm{D}}+\frac{\rho}{\ell N} \mathbf{I}+2 \mu^{k *}\left(\mathbf{S}_{\mathrm{F}}-\beta \mathbf{S}_{\mathrm{D}}\right)$ is diagonal, its pseudo-inverse can be implemented using $N$ complex multiplications. Summarizing S.1.1-S.1.3, we can conclude that the computational cost to determiner $\mathbf{c}^{k+1}$ is dominant by $\ell N$ points IFFT operation, i.e., roughly $\mathcal{O}\left(\ell N \log _{2} \ell N\right)$. Second, we analyze the computational cost to obtain $\mathrm{x}^{k+1}$. In S.2.1, the main cost to compute $\mathbf{b}^{k}$ lies in $\operatorname{IFFT}_{\ell}\left(\mathbf{c}^{k+1}\right)$. Since implementing $\ell N$ points IFFT operation needs $\frac{1}{2} \ell N \log _{2} \ell N$ complex multiplications, we can obtain $\mathbf{b}^{k}$ through roughly $\mathcal{O}\left(\ell N \log _{2} \ell N\right)$ complex multiplications. In S.2.2, Bi-section searching procedure is exploited to determine $\mathbf{z}^{k+1}$. Observing
(19), we can see that every element $z_{i}^{k+1}$ in $\mathbf{z}^{k+1}$ can be obtained just through one multiplication. Notice here $\sqrt{\frac{\alpha}{\ell N}}$ is constant and can be reused for computing all elements in $\mathbf{z}^{k+1}$. Here, it should note that the solution accuracy of the Bi-section searching procedure depends on the iteration number and the value $\gamma_{\text {right }}^{k}$ (see Table I). Usually, when it takes several iterations, for example 10, and the corresponding $\gamma_{\text {right }}^{k}=100$, pretty good solution, for example one percent accuracy in PAPR $\mathrm{dB}$ can be obtained, which is enough for the practical applications. Therefore, the computational complexity to obtain $\mathbf{z}^{k+1}$ is comparable to or less than implementing $\operatorname{IFFT}_{\ell}\left(\mathbf{c}^{k+1}\right)$, especially when $\ell$ and $N$ are large. Since the costs of implementing S.2.3 and S.2.4 are far less than that of S.2.1 and S.2.2, it also takes roughly $\mathcal{O}\left(\ell N \log _{2} \ell N\right)$ complex multiplications to compute $\mathrm{x}^{k+1}$. At last, in S.3, since $\operatorname{IFFT}_{\ell}\left(\mathbf{c}^{k+1}\right)$ is already obtained in S.2.1, $\mathbf{y}^{k+1}$ can be obtained through $\ell N$ complex multiplications. Combining the above analysis on S.1-S.3, we can conclude that the total computational cost in each ADMM-Direct iteration is the order $\mathcal{O}\left(\ell N \log _{2} \ell N\right)$.

Moreover, we should note that it may take many iterations to let ADMM-Direct converge, which could be a significant burden in practice. However, in the simulation section, we show that good OFDM symbol, i.e., with quasi-constant PAPR and very small distortion, can be obtained just after a few iterations.

2) Convergence issue: We have the following theorem on ADMM-Direct algorithm. Its proof is shown in Appendix B.

Theorem 1: Let $\left\{\mathbf{c}^{k}, \mathbf{x}^{k}, \mathbf{y}^{k}, k=1,2, \cdots\right\}$ be the tuples generated by the proposed ADMM-Direct algorithm. If $\lim \left\{\mathbf{c}^{k}, \mathbf{x}^{k}, \mathbf{y}^{k}\right\}=\left(\mathbf{c}^{*}, \mathbf{x}^{*}, \mathbf{y}^{*}\right)$, then $\left(\mathbf{c}^{*}, \mathbf{x}^{*}, \mathbf{y}^{*}\right)$ is some KKT point of the model (3).

Remarks: Here, we should note that the above Theorem 1 just states the quality of the solution when the ADMM-Direct algorithm is convergent. Exact convergence analysis is difficult since the feasible region in the model (3) is non-convex. Actually, the convergence analysis of the ADMM algorithm for the general non-convex optimization problem is still open to date. Existing analysis methods, such as in [26] and [27], cannot be exploited since the non-convex model (3) cannot satisfy their specifical conditions. However, the simulation results in this paper show that the proposed ADMM-Direct algorithm always converges, and the resulting OFDM symbols have good practical performance, i.e. quasi-constant PAPR values. In the next section, we develop a different ADMM algorithm named ADMM-Relax for the model (3), which is theoretically guaranteed to be convergent and can be arbitrarily close to some KKT point of the model (3) if proper penalty parameters are chosen.

\section{Solving Algorithm II: ADMM-Relax}

In this section, we propose the ADMM-Relax algorithm. In ADMM-Relax, we relax the model (3) to the model (21) and then we use the ADMM technique to solve the model (21). In this algorithm, all the subproblems' optimal solutions can be determined semi-analytically and the computational complexity in each iteration is roughly $\mathcal{O}\left(\ell N \log _{2} \ell N\right)$. Furthermore, 
we prove that ADMM-Relax is convergent and can approach arbitrarily close to some KKT point of the model (3) if proper parameters are chosen. Morever, the simulation results show that the optimal OFDM symbols optimized by ADMM-Relax through a few iterations have quasi-constant PAPR values.

\section{A. ADMM-Relax Algorithm Framework}

In ADMM-Relax, we relax the model (3) to the model 21 by introducing auxiliary variables $\mathbf{u}$ and $\mathbf{w}$ for the constraint (3d) and adding penalty $\|\mathbf{u}-\mathbf{w}\|_{2}^{2}$ to the objective function. The proposed ADMM-Relax algorithm is shown as follows

$$
\begin{aligned}
\min _{\mathbf{c} \in \mathbb{C}^{N}, \mathbf{x}, \mathbf{u}, \mathbf{w} \in \mathbb{C}^{\ell N}} & \frac{1}{2}\left\|\mathbf{S}_{\mathrm{D}}\left(\mathbf{c}-\mathbf{c}_{\mathrm{o}}\right)\right\|_{2}^{2}+\frac{\tilde{\rho}}{2}\|\mathbf{u}-\mathbf{w}\|_{2}^{2}, \\
\text { subject to } & \frac{\|\mathbf{x}\|_{\infty}^{2}}{\frac{1}{\ell N}\|\mathbf{x}\|_{2}^{2}}=\alpha \\
& \frac{\left\|\mathbf{S}_{\mathrm{F}} \mathbf{c}\right\|_{2}^{2}}{\left\|\mathbf{S}_{\mathrm{D}} \mathbf{c}\right\|_{2}^{2}} \leq \beta \\
& \mathbf{A c}=\mathbf{u} \\
& \mathbf{x}=\mathbf{w},
\end{aligned}
$$

where $\tilde{\rho}>0$ is the penalty factor. Intuitively, $\mathbf{u}$ and $\mathbf{w}$ can be arbitrarily close if $\tilde{\rho}$ is large enough. The augmented Lagrangian function for the model (21) is formulated as

$$
\begin{aligned}
L_{\rho}\left(\mathbf{c}, \mathbf{x}, \mathbf{u}, \mathbf{w}, \mathbf{y}_{1}, \mathbf{y}_{2}\right)=\frac{1}{2}\left\|\mathbf{S}_{\mathrm{D}}\left(\mathbf{c}-\mathbf{c}_{\mathrm{o}}\right)\right\|_{2}^{2} \\
+\operatorname{Re}\left(\mathbf{y}_{1}^{H}(\mathbf{A c}-\mathbf{u})\right)+\operatorname{Re}\left(\mathbf{y}_{2}^{H}(\mathbf{x}-\mathbf{w})\right) \\
+\frac{\tilde{\rho}}{2}\|\mathbf{u}-\mathbf{w}\|_{2}^{2}+\frac{\rho}{2}\left(\|\mathbf{A} \mathbf{c}-\mathbf{u}\|_{2}^{2}+\|\mathbf{x}-\mathbf{w}\|_{2}^{2}\right),
\end{aligned}
$$

where $\mathbf{y}_{1} \in \mathbb{C}^{\ell N}$ and $\mathbf{y}_{2} \in \mathbb{C}^{\ell N}$ are Lagrangian multipliers corresponding to the constraints (21d) and 21e respectively. The proposed ADMM-Relax algorithm for the model (3) is formulated as follows

$$
\begin{aligned}
& \mathbf{c}^{k+1}=\underset{\mathbf{c} \in \mathcal{C}}{\arg \min } L_{\rho}\left(\mathbf{c}, \mathbf{x}^{k}, \mathbf{u}^{k}, \mathbf{w}^{k}, \mathbf{y}_{1}^{k}, \mathbf{y}_{2}^{k}\right), \\
& \mathbf{x}^{k+1}=\underset{\mathbf{x} \in \mathcal{X}}{\arg \min } L_{\rho}\left(\mathbf{c}^{k+1}, \mathbf{x}, \mathbf{u}^{k}, \mathbf{w}^{k}, \mathbf{y}_{1}^{k}, \mathbf{y}_{2}^{k}\right), \\
& \left(\mathbf{u}^{k+1}, \mathbf{w}^{k+1}\right)=\underset{\mathbf{u}, \mathbf{w} \in \mathbb{C}^{\ell N}}{\arg \min } L_{\rho}\left(\mathbf{c}^{k+1}, \mathbf{x}^{k+1}, \mathbf{u}, \mathbf{w}, \mathbf{y}_{1}^{k}, \mathbf{y}_{2}^{k}\right), \\
& \mathbf{y}_{1}^{k+1}=\mathbf{y}_{1}^{k}+\rho\left(\mathbf{A} \mathbf{c}^{k+1}-\mathbf{u}^{k+1}\right), \\
& \mathbf{y}_{2}^{k+1}=\mathbf{y}_{2}^{k}+\rho\left(\mathbf{x}^{k+1}-\mathbf{w}^{k+1}\right),
\end{aligned}
$$

where $\mathrm{x} \in \mathcal{X}$ and $\mathbf{c} \in \mathcal{C}$ denote the constraints (21b) and (21c) respectively, and $k$ is the iteration number. Solving 23a and $23 \mathrm{~b}$ are quite similar to solving $4 \mathrm{a}$ and 4b. Their optimal solutions can also be determined semi-analytically. Moreover, 23c is an unconstrained convex quadratic problem. It means that its optimal solutions can also be expressed in close-form. Detailed derivations for $\left(\mathbf{c}^{k+1}, \mathbf{x}^{k+1}, \mathbf{u}^{k+1}, \mathbf{w}^{k+1}\right)$ are presented in Appendix C. In Figure 2, we summarize the proposed ADMM-Relax algorithm for the model (3).

\section{B. Performance Analysis}

1) Convergence issue: We have Theorem 2 to show the convergence properties of the proposed ADMM-Relax algorithm 23. Its proof is shown in Appendix D.
Initialization: Initialize $\left(\mathbf{c}^{1}, \mathbf{x}^{1}, \mathbf{u}^{1}, \mathbf{w}^{1}, \mathbf{y}_{1}^{1}, \mathbf{y}_{2}^{1}\right)$.

Choose parameters $(\alpha, \beta, \rho, \tilde{\rho})$. Based on the considered OFDM scheme, set diagonal matrices $\mathbf{S}_{\mathrm{D}}$ and $\mathbf{S}_{\mathrm{F}}$.

For $k=1,2,3 \cdots$

S.1 Solve the subproblem (23a).

1.1 Compute $\mathbf{v}^{k}=\mathbf{c}_{\mathrm{o}}+\frac{\rho}{\ell N} \mathrm{FFT}_{\ell}\left(\mathbf{u}^{k}-\frac{\mathbf{y}_{1}^{k}}{\rho}\right)$.

1.2 Compute

$\mu^{k *}=\max \left\{0, \frac{\left(1+\frac{\rho}{\ell N}\right)\left\|\mathbf{S}_{\mathrm{F}} \mathbf{v}^{k}\right\|_{2}-\sqrt{\beta} \frac{\rho}{\ell N}\left\|\mathbf{S}_{\mathrm{D}} \mathbf{v}^{k}\right\|_{2}}{2\left(\beta\left\|\mathbf{S}_{\mathrm{F}} \mathbf{v}^{k}\right\|_{2}+\sqrt{\beta}\left\|\mathbf{S}_{\mathrm{D}} \mathbf{v}^{k}\right\|_{2}\right)}\right\}$.

1.3 Compute

$\mathbf{c}^{k+1}=\left(\mathbf{S}_{\mathrm{D}}+\frac{\rho}{\ell N} \mathbf{I}+2 \mu^{k *}\left(\mathbf{S}_{\mathrm{F}}-\beta \mathbf{S}_{\mathrm{D}}\right)\right)^{\dagger} \mathbf{v}^{k}$.

S.2 Solve the subproblem 23b.

2.1 Compute $\mathbf{b}^{k}=\mathbf{w}^{k}-\frac{\mathbf{y}_{2}^{k}}{\rho}$.

2.2 Compute $\mathbf{z}^{k+1}$ through the binary section searching procedure in Table \

2.3 Compute $t^{k+1}=\max \left\{0, \operatorname{Re}\left(\mathbf{z}^{k+1 H} \mathbf{b}^{k}\right)\right\}$.

2.4 Compute $\mathbf{x}^{k+1}=t^{k+1} \mathbf{z}^{k+1}$.

S.3 Solve the subproblem 23c.

3.1 Compute

$\mathbf{u}^{k+1}=\frac{\mathbf{y}_{1}^{k}+\tilde{\rho} \mathbf{x}^{k+1}+(\rho+\tilde{\rho}) \operatorname{IFFT}_{\ell}\left(\mathbf{c}^{k+1}\right)}{2 \tilde{\rho}+\rho}$.

3.2 Compute

$\mathbf{w}^{k+1}=\frac{\mathbf{y}_{2}^{k}+(\tilde{\rho}+\rho) \mathbf{x}^{k+1}+\tilde{\rho} \operatorname{IFFT}_{\ell}\left(\mathbf{c}^{k+1}\right)}{2 \tilde{\rho}+\rho}$.

S.4 Update Lagrangian multipliers.

4.1 Compute $\mathbf{y}_{1}^{k+1}=\mathbf{y}_{1}^{k}+\rho\left(\operatorname{IFFT}_{\ell}\left(\mathbf{c}^{k+1}\right)-\mathbf{u}^{k+1}\right)$.

4.2 Compute $\mathbf{y}_{2}^{k+1}=\mathbf{y}_{2}^{k}+\rho\left(\mathbf{x}^{k+1}-\mathbf{w}^{k+1}\right)$.

Until some preset termination conditions are satisfied.

Then, let $\mathrm{x}^{k+1}$ be the output.

Fig. 2: ADMM-Relax algorithm for the model (3).

Theorem 2: Let $\left\{\mathbf{c}^{k}, \mathbf{x}^{k}, \mathbf{u}^{k}, \mathbf{w}^{k}, \mathbf{y}_{1}^{k}, \mathbf{y}_{2}^{k}, k=1,2, \cdots\right\}$ be the sequence generated by the proposed ADMM-Relax algorithm (23) as shown in Figure 2. If $\rho>2 \tilde{\rho}$, then

- sequence $\left\{\mathbf{c}^{k}, \mathbf{x}^{k}, \mathbf{u}^{k}, \mathbf{w}^{k}, \mathbf{y}_{1}^{k}, \mathbf{y}_{2}^{k}\right\}$ is convergent, i.e.,

$$
\begin{array}{ll}
\lim _{k \rightarrow+\infty} \mathbf{c}^{k}=\mathbf{c}^{*}, & \lim _{k \rightarrow+\infty} \mathbf{x}^{k}=\mathbf{x}^{*}, \\
\lim _{k \rightarrow+\infty} \mathbf{u}^{k}=\mathbf{u}^{*}, & \lim _{k \rightarrow+\infty} \mathbf{w}^{k}=\mathbf{w}^{*}, \\
\lim _{k \rightarrow+\infty} \mathbf{y}_{1}^{k}=\mathbf{y}_{1}^{*}, & \lim _{k \rightarrow+\infty} \mathbf{y}_{2}^{k}=\mathbf{y}_{2}^{*},
\end{array}
$$

and $\mathbf{A c}^{*}=\mathbf{u}^{*}, \mathbf{x}^{*}=\mathbf{w}^{*}, \mathbf{y}_{1}^{*}=-\mathbf{y}_{2}^{*}$.

- $\left(\mathbf{c}^{*}, \mathbf{x}^{*}, \mathbf{u}^{*}, \mathbf{w}^{*}\right)$ is some KKT point of the model (21).

- If $\left(\mathbf{c}^{1}, \mathbf{x}^{1}\right)$ lies in the feasible region of the model (3), $\left(\mathbf{c}^{*}, \mathbf{x}^{*}\right)$ approaches some KKT point of the model (3) as $\tilde{\rho}$ increases.

Remarks: Theorem 2 shows that the proposed ADMMRelax algorithm is theoretically guaranteed to be convergent. Especially, its third part indicates that if $\left(\mathbf{c}^{1}, \mathbf{x}^{1}\right)$ lies in the feasible region of the original model (3), $\left(\mathbf{c}^{*}, \mathbf{x}^{*}\right)$ approaches some KKT point of the model (3) as $\tilde{\rho}$ increases. Moreover, in the simulation section, we also show that the residual error, $\left\|\mathbf{A} \mathbf{c}^{*}-\mathbf{x}^{*}\right\|_{2}^{2}$, of the KKT equations decreases as $\tilde{\rho}$ increases. Furthermore, the key to prove Theorem 2 is to exploit the 
unconstrained auxiliary variables $\mathbf{u}$ and $\mathbf{w}$, the augmented Lagrangian function can be guaranteed sufficient descent in every iteration. However, in ADMM-Direct, the corresponding augmented Lagrangian function cannot be proved to have this kind of property. The detailed proof of Theorem 2 can be found in Appendix B.

Moreover, the relaxation does not cause larger PAPR values than $\alpha$ since we let the optimized $\mathbf{x}$ be the final output. In the above theorem, we mention that, to guarantee the convergence of ADMM-Relax, $\rho$ and $\tilde{\rho}$ should satisfy $\rho>2 \tilde{\rho}>0$. Besides that, we should note that there is no theoretical results to help us to set their values. However, it can be seen that the relaxed optimization problem (21) could be ill-conditional if $\tilde{\rho}$ is too large. In the classical augmented Lagrangian multiplier method, there is similar problem on how to set penalty factor. In practice, a general way, but heuristic, to choose proper $\tilde{\rho}$ is to perform simulations when different $\tilde{\rho}$ are set and then select the value corresponding to the best simulation result. In the simulation section, we choose $\tilde{\rho}=100$ and $\rho=300$, which leads to pretty good optimization results.

2) Iteration complexity: We use the residual error which is defined as $\left\|\mathbf{u}^{k+1}-\mathbf{u}^{k}\right\|_{2}^{2}+\left\|\mathbf{w}^{k+1}-\mathbf{w}^{k}\right\|_{2}^{2}$ to measure the convergence progress of the ADMM-Relax algorithm since it converges to zero as $k \rightarrow+\infty$. Then, we have Theorem 3 about its convergence progress. The detailed proof is shown in Appendix E.

Theorem 3: Let $r$ be the minimum iteration index such that $\left\|\mathbf{u}^{k+1}-\mathbf{u}^{k}\right\|_{2}^{2}+\left\|\mathbf{w}^{k+1}-\mathbf{w}^{k}\right\|_{2}^{2} \leq \epsilon$, where $\epsilon$ is the desired precise parameter for the solution. Then, we have the following iteration complexity result

$$
\begin{aligned}
r \leq \frac{1}{C \epsilon}( & L_{\rho}\left(\mathbf{c}^{1}, \mathbf{x}^{1}, \mathbf{u}^{1}, \mathbf{w}^{1}, \mathbf{y}_{1}^{1}, \mathbf{y}_{2}^{1}\right) \\
& \left.-\left(\frac{1}{2}\left\|\mathbf{S}_{\mathrm{D}}\left(\mathbf{c}^{*}-\mathbf{c}_{\mathrm{o}}\right)\right\|_{2}^{2}+\frac{\tilde{\rho}}{2}\left\|\mathbf{u}^{*}-\mathbf{w}^{*}\right\|_{2}^{2}\right)\right),
\end{aligned}
$$

where $\rho>2 \tilde{\rho}$ and the constant $C$ is the minimum eigenvalue of the following positive definite matrix

$$
\left[\begin{array}{cc}
\frac{\tilde{\rho}+\rho}{2}-\frac{2 \tilde{\rho}^{2}}{\rho} & \frac{2 \tilde{\rho}^{2}}{\rho}-\frac{\tilde{\rho}}{2} \\
\frac{2 \tilde{\rho}^{2}}{\rho}-\frac{\tilde{\rho}}{2} & \frac{\tilde{\rho}+\rho}{2}-\frac{2 \tilde{\rho}^{2}}{\rho}
\end{array}\right] .
$$

3) Computational cost: In Figure 2, we still use operators $\operatorname{IFFT}_{\ell}(\cdot)$ and $\operatorname{FFT}_{\ell}(\cdot)$ to take the place of $\mathbf{A}$ and $\mathbf{A}^{H}$ respectively. Similar to the computational complexity analysis for ADMM-Direct, we can conclude that the computational cost in each ADMM-Relax iteration is roughly $\mathcal{O}\left(\ell N \log _{2} \ell N\right)$. Combining this result with Theorem 3 , we conclude that the total computational cost to attain an $\epsilon$-optimal solution is $\mathcal{O}\left(\lfloor d\rfloor \ell N \log _{2} \ell N\right)$, where $d=$ $\frac{1}{C \epsilon}\left(L_{\rho}\left(\mathbf{c}^{1}, \mathbf{x}^{1}, \mathbf{u}^{1}, \mathbf{w}^{1}, \mathbf{y}_{1}^{1}, \mathbf{y}_{2}^{1}\right)-\left(\frac{1}{2}\left\|\mathbf{S}_{\mathrm{D}}\left(\mathbf{c}^{*}-\mathbf{c}_{\mathrm{o}}\right)\right\|_{2}^{2}+\frac{\tilde{\rho}}{2} \| \mathbf{u}^{*}-\right.\right.$ $\left.\left.\mathbf{w}^{*} \|_{2}^{2}\right)\right)$.

\section{Simulation Results}

In this section, several simulation results are presented to illustrate the performance of the proposed ADMM-Direct and ADMM-Relax. We compare the proposed algorithms with the RCF approach [10], Aggarwal SOCP approach [14], simplified OICF approach [17], modified SLM approach [28] and lowcomplexity tone injection scheme [29].
Throughout this section, simulation parameters are set as follows: PAPR constraint, for ADMMs, is $4.0 \mathrm{~dB}$. Free carrier power overhead, for ADMMs, is $0,0.15$, and 0.3 . The penalty parameters for ADMM-Direct are $\rho=100$, and for ADMMRelax they are $\rho=300$ and $\tilde{\rho}=100$. Consider an OFDM scheme 3 with 52 data carriers and 12 free carriers and the over-sampling factor $\ell=4$. For all of the bit error ratio (BER) simulations, we calculate $\mathrm{E}_{\mathrm{b}}$ by

$$
\mathrm{E}_{\mathrm{b}}=\frac{\overline{\mathrm{E}}_{\mathrm{s}}}{\mathrm{M} \cdot \bmod \text { _style }},
$$

where $\overline{\mathrm{E}}_{\mathrm{S}}$ represents the averaged energy of the optimal frequency-domain OFDM symbols, $M$ is the number of data carriers, and "mod_style" represents the modulation scheme, which is 2 and 4 corresponding to QPSK and 16-QAM modulation respectively. In the simulations, the number of OFDM symbols are 5000. Moreover, all the simulations are implemented in MATLAB 2017 environment.

Figure 3 shows the convergence curves of the proposed ADMM-Direct and ADMM-Relax algorithms. In Figure 3 (a), the residual error of ADMM-Direct is defined as $\| \mathbf{c}^{k+1}-$ $\mathbf{c}^{k}\left\|_{2}^{2}+\right\| \mathbf{x}^{k+1}-\mathbf{x}^{k} \|_{2}^{2}$, and in Figure 3 (b), the residual error of ADMM-Relax is defined as $\left\|\mathbf{u}^{k+1}-\mathbf{u}^{k}\right\|_{2}^{2}+\left\|\mathbf{w}^{k+1}-\mathbf{w}^{k}\right\|_{2}^{2}$. From the curves, we can see that both of the ADMMs can converge after a few iterations. Here, we should note that we do not give the exact proof of the convergence for the ADMM-Direct algorithm. However, we observe that ADMMDirect can converge from Figure 3(a). From Figure 3 (b), we can see that the residual error decreases quickly in the first several iterations and after 5 iterations, the convergence curve is relatively flat.

To further illustrate the third part of Theorem 2] which indicates that if $\left(\mathbf{c}^{1}, \mathbf{x}^{1}\right)$ lies in the feasible region of the model (3), $\left(\mathbf{c}^{*}, \mathbf{x}^{*}\right)$ approaches some KKT point of the model (3) as $\tilde{\rho}$ increases, we plot the curve of the relationship between $\left\|\mathbf{A} \mathbf{c}^{*}-\mathbf{x}^{*}\right\|_{2}^{2}$ and $\tilde{\rho}$ in Figure 4 In this figure, $\left(\mathbf{c}^{*}, \mathbf{x}^{*}\right)$ is the optimal solution of the model (21). Moreover, we use $\left\|\mathbf{A} \mathbf{c}^{*}-\mathbf{x}^{*}\right\|_{2}^{2}$ to measure whether $\left(\mathbf{c}^{*}, \mathbf{x}^{*}\right)$ approaches some KKT point of the model (3), because if $\left\|\mathbf{A} \mathbf{c}^{*}-\mathbf{x}^{*}\right\|_{2}^{2}$ approaches zero, $\left(\mathbf{c}^{*}, \mathbf{x}^{*}\right)$ approaches some KKT point of the model (3) as Appendix D shows. From the curve, we can see that, $\left\|\mathbf{A} \mathbf{c}^{*}-\mathbf{x}^{*}\right\|_{2}^{2}$ approaches zero as $\tilde{\rho}$ increases as we expected. That is, $\left(\mathbf{c}^{*}, \mathbf{x}^{*}\right)$ approaches some KKT point of (3).

In Table II, we show that the impact of the different values of $\beta$ on signal distortion introduced to data carriers of OFDM signals. Here, we use averaged error vector magnitude (EVM) to evaluate distortion, which is defined by

$$
\overline{\mathrm{EVM}}=\sqrt{\frac{1}{K} \sum_{i=1}^{K} \frac{\left\|\mathbf{S}_{\mathrm{D}}\left(\mathbf{c}-\mathbf{c}_{\mathrm{o}}\right)\right\|_{2}^{2}}{\left\|\mathbf{c}_{\mathrm{o}}\right\|_{2}^{2}}}
$$

\footnotetext{
${ }^{3}$ The considered OFDM scheme is based on IEEE 802.11a/g Wi-Fi standard. The proposed ADMM-Direct/-Relax algorithms can also be applied to reducing PAPR values of OFDM signals in the $4 \mathrm{G} / 5 \mathrm{G}$ cellular systems. But the simulation parameters, such as $\alpha, \beta, \rho$, and $\tilde{\rho}$, should be re-chosen carefully to achieve desired system performance. Moreover, since the proposed algorithms have much cheaper computational complexity in each iteration than state-of-the-art PAPR reduction approaches, they could be more suitable for large-scale OFDM system.
} 


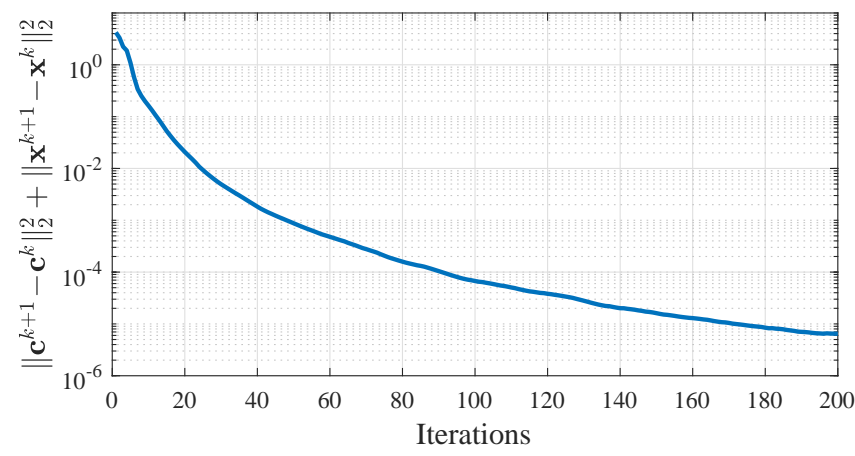

(a) ADMM-Direct

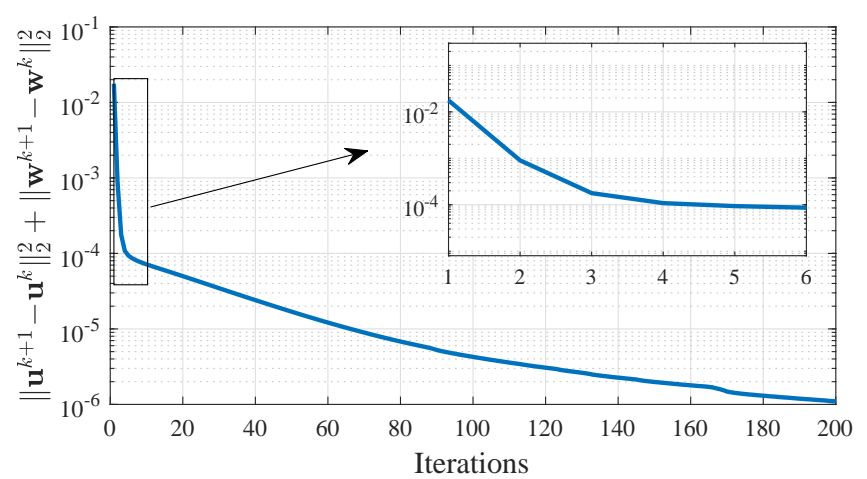

(b) ADMM-Relax

Fig. 3: The convergence performance of ADMM-Direct and ADMM-Relax with 16-QAM modulation.

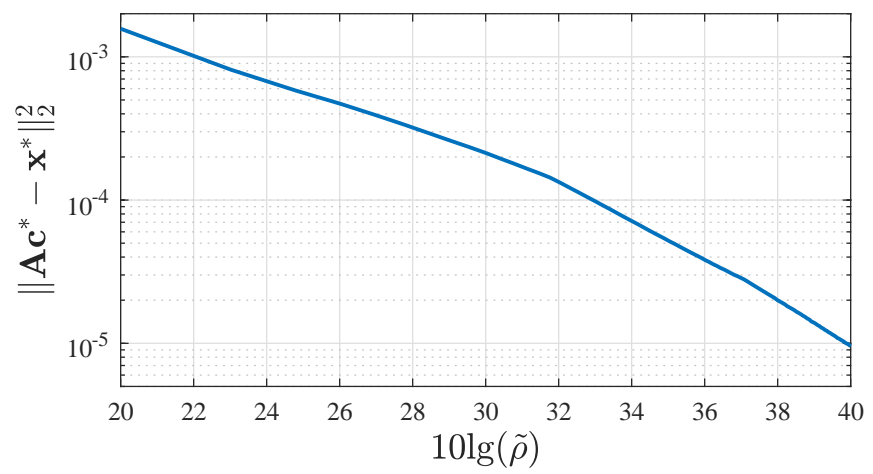

Fig. 4: The relationship between $\left\|\mathbf{A} \mathbf{c}^{*}-\mathbf{x}^{*}\right\|_{2}^{2}$ and $\tilde{\rho}$.

TABLE II: Comparison of EVMs (dB) at different $\beta$ (Modulation: 16-QAM; PAPR constraint: 4dB)

\begin{tabular}{|c||c|c|}
\hline \hline \multirow{2}{*}{\multicolumn{1}{|c||}{$\beta$}} & \multicolumn{2}{c|}{ EVM } \\
\cline { 2 - 3 } & ADMM-Direct & ADMM-Relax \\
\hline 0 & $-16.58 \mathrm{~dB}$ & $-16.36 \mathrm{~dB}$ \\
\hline 0.15 & $-27.33 \mathrm{~dB}$ & $-27.51 \mathrm{~dB}$ \\
\hline 0.3 & $-32.96 \mathrm{~dB}$ & $-32.89 \mathrm{~dB}$ \\
\hline \hline
\end{tabular}

where $K$ is set 5000 in the simulations. From Table II, we can see clearly that the signal distortion decreases almost $10 \mathrm{~dB}$ when we increase $\beta$ from 0 to 0.15 . However, when we further increase $\beta$ from 0.15 to 0.3 , the corresponding EVM values, i.e., introduced distortions, only decrease about $5.5 \mathrm{~dB}$. Checking the optimized free carriers, we find that the power overheads of some OFDM symbols in the latter are less than the pre-set upper-bound 0.3. It means that the constraint 3(c) becomes inactive for these kinds of OFDM symbols and their optimizers locate inside the defined feasible region. The fact indicates that increasing $\beta$ can decrease averaged signal distortion of the optimized OFDM symbols efficiently. However, for a larger $\beta$, its influence becomes weaker.

Figure 5 and Figure 6 plot PAPR complementary cumulative distribution functions (CCDFs), bit error rate (BER) performance of the original and processed OFDM signals after the solid state PA (SSPA) with smoothing factor 3 (modeled in [3]) and through AWGN channel, and BER performance of OFDM signals after SSPA and through multipath channel. Data carrier modulations are assumed to be 16QAM or QPSK. CCDF denotes the probability that the PAPR of the OFDM symbols exceeds some given threshold $T$, i.e., $\operatorname{CCDF}(T)=\operatorname{Prob}(\mathrm{PAPR}>T)$. The simulation results of ADMMs are obtained through 5 iterations.

In Figure 5(a), the word "Original" means no distortion is introduced. From the figure, we can see that the proposed ADMMs, RCF, OICF, and SDP, have cut-off CCDF curves. However, the CCDF curves of ADMMs and SDP locate on the left side of the others'. It means that the former three approaches have better PAPR reduction performance. Moreover, CCDF curves of SOCP, modified SLM and tone injection are slow-down. It means that some of their optimized OFDM symbols still have larger PAPR values. In practice, these kinds of signals would suffer from severe nonlinear distortion of the PA, which can worse BER performance of the OFDM signals. Figure 5(b) shows the BER curves of the optimized OFDM symbols after SSPA. The input power backoff of the working-point away from saturation region is set as $4.1 \mathrm{~dB}$. From it, we can see that BER curves of ADMMs and SDP are closest to the ideal's. Here, the word "ideal" means no distortion is introduced in the OFDM symbols. Figure 6 plots the PAPR-reduction performance and BER performance of the original OFDM signals and the processed OFDM signals with QPSK data carrier modulation. Similar to the performance with 16-QAM modulation, ADMMDirect and ADMM-Relax still have cut-off PAPR reduction performance. Meanwhile, from Figure 6(b), we can see that the ADMM-Direct and ADMM-Relax have pretty good BER performance. Here, the input power back-off is also set as $4.1 \mathrm{~dB}$. Furthermore, Figure 5(c) and 6 (c) plot the BER curves of the optimized OFDM symbols after SSPA and multi-path channel. Here, four paths are considered and in each path delay/fading parameters are $(0,1)$ (direct path), $(190,0.2)$, $(300,0.07)$, and $(400,0.05)$ respectively. From the figures, we can see that, in comparison with AWGN channel case, BER performance of all PAPR reduction methods becomes worse when multi-path effects are considered. However, ADMMDirect/-Relax approaches are still better than state-of-the-art 


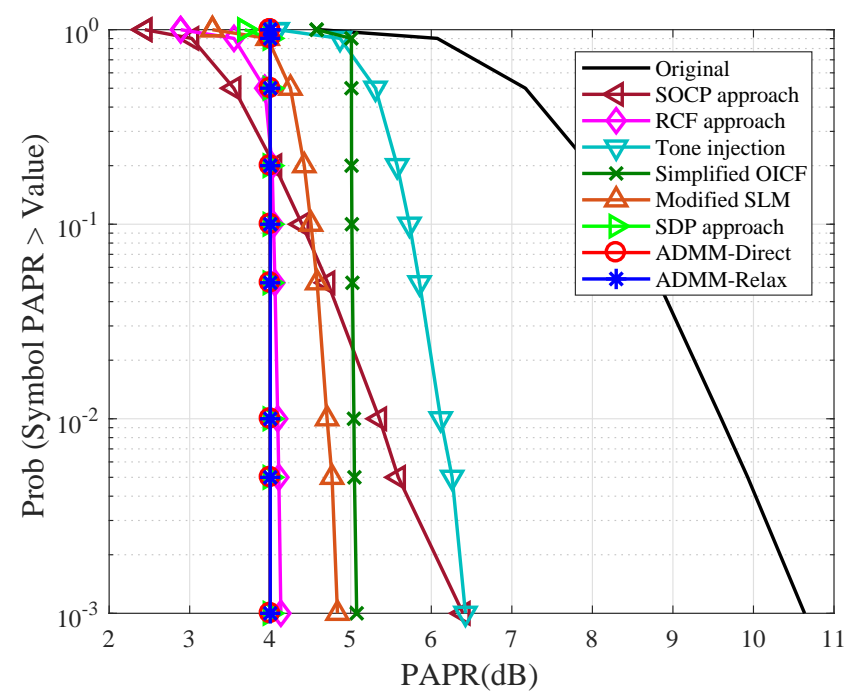

(a) PAPR reduction performance

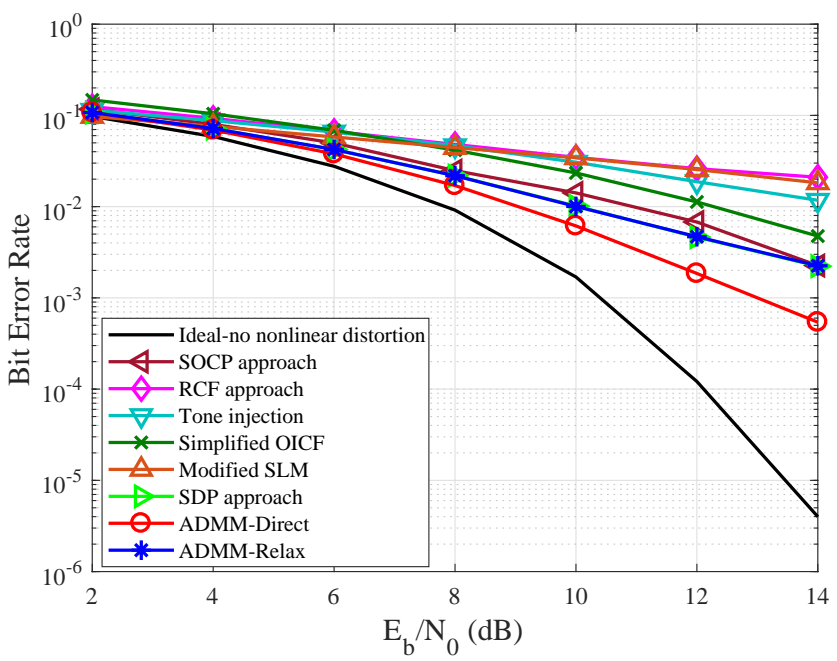

(b) BER performance (after SSPA)

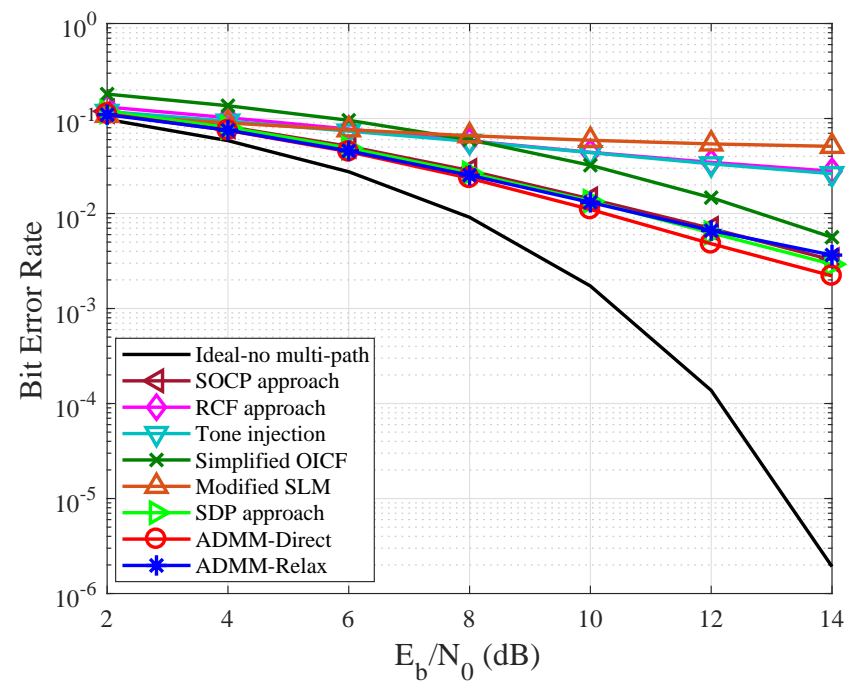

(b) BER performance (through multi-path channel)

Fig. 5: The performance of various systems with 16-QAM modulation(5 iterations for ADMMs, 10 iterations for RCF). approaches. At last, from Figure 5 and 6, we see that SDP approach has similar PAPR reduction performance and BER performance to our proposed ADMM approaches. However, we should note that the computational complexity of SDP is prohibitive in practice, which is roughly $\mathcal{O}\left(\ell^{3} N^{3}\right)$ in each iteration. In comparison, the computational complexity of our ADMMs is roughly $\mathcal{O}\left(\ell N \log _{2}(\ell N)\right)$ in each iteration, which is much cheaper than SDP. The out-of-band radiation performance is shown in Figure 7 On the one hand, we can see from the curves that SDP, ADMM-Direct/-Relax and RCF have lower out-of-band emission (OOBE). On the other hand, we can also observe that tone injection and modified SLM have higher OOBE. The reason that OOBE performance of the proposed ADMM-Direct/-Relax approaches and SDP appraoch is better because all of them have cut-off CCDF curves, which means that their processed OFDM symbols have quasi-constant PAPR values. It is well known that OOBE is mainly caused by nonlinear distortion of the OFDM signals. In the simulations, since the input power back-off of the working-point is set as $4.1 \mathrm{~dB}$ and the PAPR of OFDM symbols optimized by ADMM-Direct/-Relax are almost 4dB (quasiconstant), it means that most of the signals are amplified in the linear region of the PA. So, there is only very small nonlinear distortion (caused by nonlinear PA) introduced into the OFDM symbols. Accordingly, it is reasonable that their OOBEs are low.

\section{CONCLUSION}

This paper proposes two low-complexity iteration optimization methods named ADMM-Direct and ADMM-Relax, to reduce PAPR values of the OFDM signals. Both of their computational complexities in each iteration are similar to the classical RCF method and the desired OFDM symbols can be obtained just after a few iterations; meanwhile, they have quasi-constant PAPR values and optimized signal distortion. Moreover, the resulting solution of ADMM-Direct is guaranteed to be some KKT point of the established model when the iteration algorithm converges. We also prove that ADMMRelax is convergent and can approach arbitrarily close to some KKT point of the model if proper algorithm parameters are chosen. In comparison with existing algorithms, the proposed ADMM algorithms outperform the existing approaches not only in the simulation results but also in strong theoretically guaranteed performance. In the end, we should mention that high PAPR problem is still an issue in the systems of multipleinput multiple-output (MIMO) and non-orthogonal multiple access (NOMA) [30] when multi-carriers techniques are applied. Designing low-complexity, but theoretically guaranteed, ADMM-like optimization algorithm could be an interesting research topic in the future.

\section{APPENDIX A}

\section{PROOF OF EQUiVALENCE BETWEEN MODELS (15) AND (16)}

The equivalence between the models (15) and 16) is in the sense that the global optimal solution of the latter model is always attained when "=" holds in 16c. We prove this fact by contradiction. To be clear, we suppose some feasible 


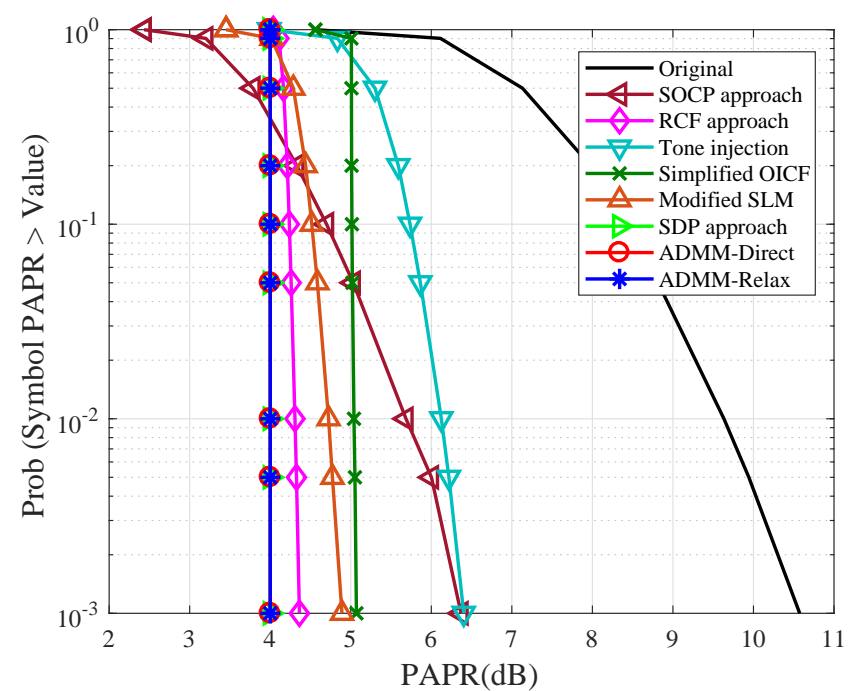

(a) PAPR reduction performance

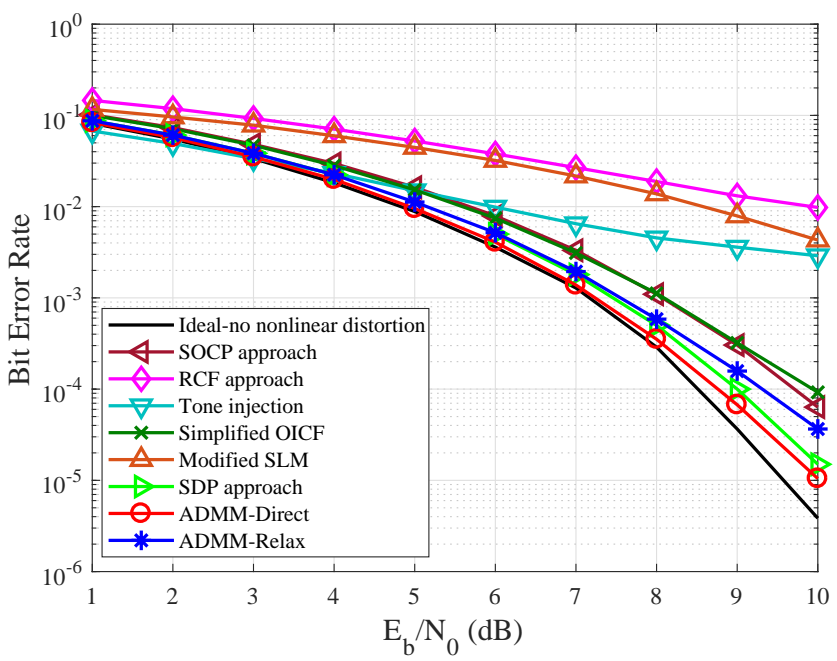

(b) BER performance (after SSPA)

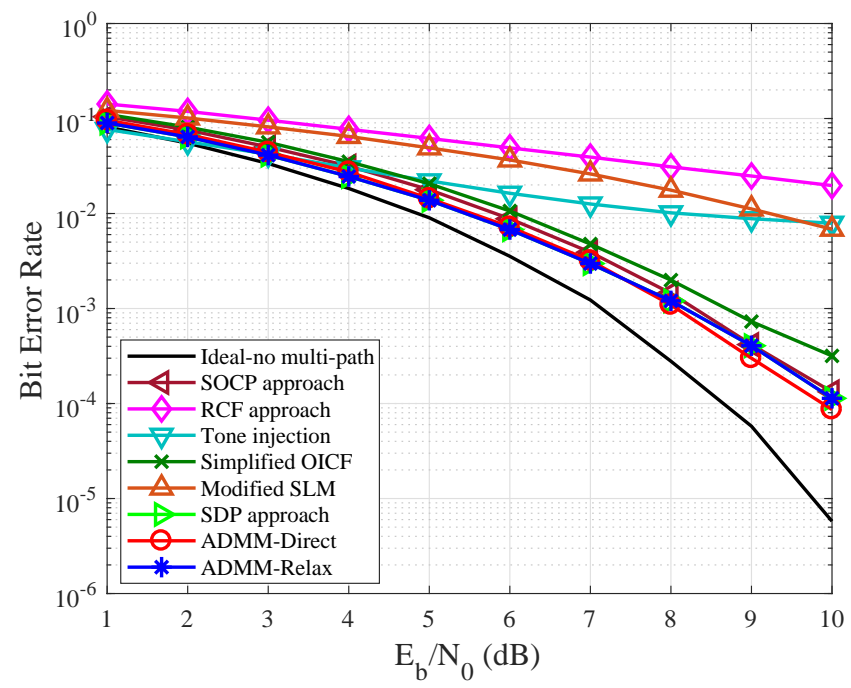

(b) BER performance (through multi-path channel)

Fig. 6: The performance of various systems with QPSK modulation(5 iterations for ADMMs, 10 iterations for RCF).

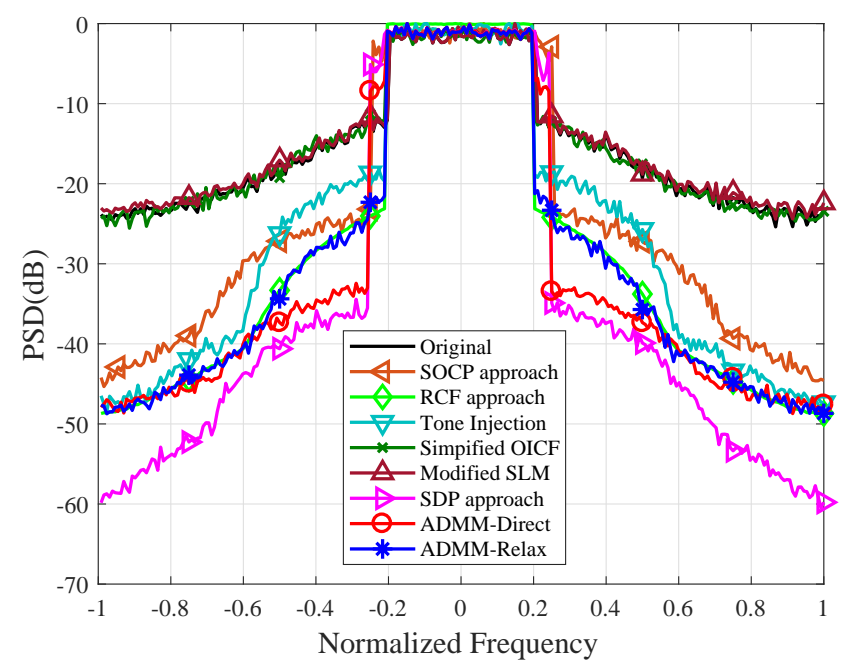

Fig. 7: Out-of-band radiation performance with 16-QAM modulation.

point $\tilde{\mathbf{z}}$ satisfies $\|\tilde{\mathbf{z}}\|_{2}^{2}<1$ and the constraint (16b). Notice that the constant modulus $\tilde{\mathbf{z}}$ is in the feasible region since $\alpha$ is greater than 1 . Therefore, we can always find a vector $\triangle \tilde{\mathbf{z}}$, whose phase vector is equal to that of vector $\mathbf{b}^{k}$, which can let $\tilde{\mathbf{z}}+\triangle \tilde{\mathbf{z}}$ satisfy the constraints 16b and 16c. It is obvious that the new vector corresponds to a larger objective value, which means that $\tilde{\mathbf{z}}$ is not the global optimal solution of the model (16). So, we can conclude that the maximizer in model (16) should always satisfy (16c) when "=" holds.

\section{APPENDIX B \\ PROOF OF THEOREM 1}

To be clear, we let $L(\mathbf{c}, \mathbf{x}, \gamma, \mu, \mathbf{y}), L^{\mathbf{c}}(\mathbf{c}, \mathbf{y}, \mu)$, and $L^{\mathbf{x}}(\mathbf{x}, \mathbf{y}, \gamma)$ be the Lagrangian functions of the model (3), 4a), and (4b) respectively. $\mu$ and $\gamma$ are the Lagrangian multipliers corresponding to the constraints (3c) and (3b) respectively. We also let $\mu^{*}$ and $\gamma^{*}$ be the corresponding optimal Lagrangian multipliers when ADMM-Direct algorithm is convergent. To show $\left(\mathbf{c}^{*}, \mathbf{x}^{*}\right)$ is a KKT point, we should prove that it, combining $\left(\mathbf{y}^{*}, \mu^{*}, \gamma^{*}\right)$, should satisfy the conditions of the primal feasibility (25a), the dual feasibility (25b) and the complementary slackness (25c), and is also a stationary point of the Lagrangian function $L(\mathbf{c}, \mathbf{x}, \gamma, \mu, \mathbf{y})$, i.e.,

$$
\begin{aligned}
& \mathbf{c}^{*} \in \mathcal{C}, \quad \mathbf{x}^{*} \in \mathcal{X}, \\
& \mu^{*} \geq 0, \\
& \mu^{*}\left(\left\|\mathbf{S}_{\mathrm{F}} \mathbf{c}^{*}\right\|_{2}^{2}-\beta\left\|\mathbf{S}_{\mathrm{D}} \mathbf{c}^{*}\right\|_{2}^{2}\right)=0, \\
& \nabla_{\mathbf{c}} L\left(\mathbf{c}^{*}, \mathbf{x}^{*}, \gamma^{*}, \mu^{*}, \mathbf{y}^{*}\right)=0 \\
& \nabla_{\mathbf{x}} L\left(\mathbf{c}^{*}, \mathbf{x}^{*}, \gamma^{*}, \mu^{*}, \mathbf{y}^{*}\right)=0
\end{aligned}
$$

where $\mathcal{X}$ and $\mathcal{C}$ denote the constraints $3 \mathrm{~b}$ and $3 \mathrm{c}$ respectively.

Since in every ADMM-Direct iteration, $\mathbf{c}^{k+1}$ and $\mathbf{x}^{k+1}$ are located in the feasible region, we can see that the primal feasibility condition 25a is satisfied. Since $\mu^{k *}$ is guaranteed to be greater than zero (see (12) ) in every iteration, it means 
that $\mu^{*}$ is also greater than zero. Moreover, checking (12) again, we see that the value of $\mu^{k *}, \forall k$, is nonzero or zero corresponding to the constraint $3 \mathrm{c}$, which is active or inactive respectively. It means that $\mu^{*}$ satisfies the condition of complementary slackness.

Now, let us consider 25d) and 25e. Since $\mathbf{c}^{k+1}$ and $\mathbf{x}^{k+1}$ are the minimizers of the problems (4a) and 4b in the $k$ th iteration respectively, they should satisfy

$$
\begin{aligned}
& \nabla_{\mathbf{c}} L^{\mathbf{c}}\left(\mathbf{c}^{k+1}, \mathbf{y}^{k}, \mu^{k *}\right)+\rho \mathbf{A}^{H}\left(\mathbf{A} \mathbf{c}^{k+1}-\mathbf{x}^{k}\right)=0, \\
& \nabla_{\mathbf{x}} L^{\mathbf{x}}\left(\mathbf{x}^{k+1}, \mathbf{y}^{k}, \gamma^{k *}\right)-\rho\left(\mathbf{A} \mathbf{c}^{k+1}-\mathbf{x}^{k+1}\right)=0 .
\end{aligned}
$$

Since $\lim _{k \rightarrow+\infty}\left(\mathbf{c}^{k}, \mathbf{x}^{k}, \mathbf{y}^{k}\right)=\left(\mathbf{c}^{*}, \mathbf{x}^{*}, \mathbf{y}^{*}\right)$ and $\mathbf{y}^{k+1}=\mathbf{y}^{k}+$ $\rho\left(\mathbf{A} \mathbf{c}^{k+1}-\mathbf{x}^{k+1}\right)$, we can drop the second terms in 26) as $k \rightarrow+\infty$ and obtain

$$
\nabla_{\mathbf{c}} L^{\mathbf{c}}\left(\mathbf{c}^{*}, \mathbf{y}^{*}, \mu^{*}\right)=0, \quad \nabla_{\mathbf{x}} L^{\mathbf{x}}\left(\mathbf{x}^{*}, \mathbf{y}^{*}, \gamma^{*}\right)=0 .
$$

Since there are $\nabla_{\mathbf{c}} L^{\mathbf{c}}\left(\mathbf{c}^{*}, \mathbf{y}^{*}, \mu^{*}\right)=\nabla_{\mathbf{c}} L\left(\mathbf{c}^{*}, \mathbf{x}^{*}, \gamma^{*}, \mu^{*}, \mathbf{y}^{*}\right)$ and $\nabla_{\mathbf{x}} L^{\mathbf{x}}\left(\mathbf{x}^{*}, \mathbf{y}^{*}, \gamma^{*}\right)=\nabla_{\mathbf{x}} L\left(\mathbf{c}^{*}, \mathbf{x}^{*}, \gamma^{*}, \mu^{*}, \mathbf{y}^{*}\right)$, we can see that $\left(\mathbf{c}^{*}, \mathbf{x}^{*}, \mathbf{y}^{*}\right)$ should satisfy 25d) and 25e, i.e., it is a stationary point of the Lagrangian function $L(\mathbf{c}, \mathbf{x}, \gamma, \mu, \mathbf{y})$. This concludes the proof of Theorem 1 .

\section{APPENDIX C}

\section{SOLVING THE OPTIMIZATION SUBPROBLEMS $(23 \mathrm{a})-(23 \mathrm{c}$}

\section{A. Solving the Subproblem 23a}

Based on the augmented Lagrangian function (22), the problem 23a is equivalent to

$$
\begin{aligned}
& \underset{\mathbf{c} \in \mathbb{C}^{N}}{\min } \frac{1}{2}\left\|\mathbf{S}_{\mathrm{D}}\left(\mathbf{c}-\mathbf{c}_{\mathrm{o}}\right)\right\|_{2}^{2}+\frac{\rho}{2}\left\|\mathbf{A} \mathbf{c}-\mathbf{u}^{k}+\frac{\mathbf{y}_{1}^{k}}{\rho}\right\|_{2}^{2}, \\
& \text { subject to }\left\|\mathbf{S}_{\mathrm{F}} \mathbf{c}\right\|_{2}^{2}-\beta\left\|\mathbf{S}_{\mathrm{D}} \mathbf{c}\right\|_{2}^{2} \leq 0 .
\end{aligned}
$$

Its Lagrangian function can be written as

$$
\begin{aligned}
L\left(\mathbf{c}, \mu^{k}\right)= & \frac{1}{2}\left\|\mathbf{S}_{\mathrm{D}}\left(\mathbf{c}-\mathbf{c}_{\mathrm{o}}\right)\right\|_{2}^{2}+\frac{\rho}{2}\left\|\mathbf{A} \mathbf{c}-\mathbf{u}^{k}+\frac{\mathbf{y}_{1}^{k}}{\rho}\right\|_{2}^{2} \\
& +\mu^{k}\left(\left\|\mathbf{S}_{\mathrm{F}} \mathbf{c}\right\|_{2}^{2}-\beta\left\|\mathbf{S}_{\mathrm{D}} \mathbf{c}\right\|_{2}^{2}\right),
\end{aligned}
$$

where the Lagrangian multiplier is $\mu^{k} \geq 0$. Since the global optimal solution $\mathbf{c}^{k+1}$, combining the optimal Lagrangian multiplier $\mu^{k *}$ should satisfy $\nabla_{\mathbf{c}} L\left(\mathbf{c}^{k+1}, \mu^{k *}\right)=0$, we can get

$$
\mathbf{c}^{k+1}=\left(\mathbf{S}_{\mathrm{D}}+\frac{\rho}{\ell N} \mathbf{I}+2 \mu^{k *}\left(\mathbf{S}_{\mathrm{F}}-\beta \mathbf{S}_{\mathrm{D}}\right)\right)^{\dagger} \mathbf{v}^{k},
$$

where $\mathbf{v}^{k}=\mathbf{c}_{\mathrm{o}}+\rho \mathbf{A}^{H}\left(\mathbf{u}^{k}-\frac{\mathbf{y}_{1}^{k}}{\rho}\right)$.

Moreover, when the constraint $28 \mathrm{~b}$ is inactive, $\mathbf{c}^{k+1}$ is located inside the feasible region $\mathcal{C}$. It means that $\mu^{k *}=0$. Otherwise, if the constraint 28b is active, it means that $\mathbf{c}^{k+1}$ and the optimal Lagrangian multiplier $\mu^{k *}$ should satisfy the constraint 28b when "=" holds. Plugging 30 into $\left\|\mathbf{S}_{\mathrm{F}} \mathbf{c}^{k+1}\right\|_{2}$ and $\left\|\mathbf{S}_{\mathrm{D}} \mathbf{c}^{k+1}\right\|_{2}$, we can obtain

$$
\begin{aligned}
\left\|\mathbf{S}_{\mathrm{F}} \mathbf{c}^{k+1}\right\|_{2} & =\frac{\left\|\mathbf{S}_{\mathrm{F}} \mathbf{v}^{k}\right\|_{2}}{\frac{\rho}{\ell N}+2 \mu^{k *}}, \\
\left\|\mathbf{S}_{\mathrm{D}} \mathbf{c}^{k+1}\right\|_{2} & =\frac{\left\|\mathbf{S}_{\mathrm{D}} \mathbf{v}^{k}\right\|_{2}}{1+\frac{\rho}{\ell N}-2 \mu^{k *} \beta} .
\end{aligned}
$$

Then, we can solve $\mu^{k *}$ as (32) when $\left\|\mathbf{S}_{\mathrm{F}} \mathbf{c}^{k+1}\right\|_{2}^{2}=$ $\beta\left\|\mathbf{S}_{\mathrm{D}} \mathbf{c}^{k+1}\right\|_{2}^{2}$.

$$
\mu^{k *}=\frac{\left(1+\frac{\rho}{\ell N}\right)\left\|\mathbf{S}_{\mathrm{F}} \mathbf{v}^{k}\right\|_{2}-\sqrt{\beta} \frac{\rho}{\ell N}\left\|\mathbf{S}_{\mathrm{D}} \mathbf{v}^{k}\right\|_{2}}{2\left(\beta\left\|\mathbf{S}_{\mathrm{F}} \mathbf{v}^{k}\right\|_{2}+\sqrt{\beta}\left\|\mathbf{S}_{\mathrm{D}} \mathbf{v}^{k}\right\|_{2}\right)} .
$$

Furthermore, observing (32), we see that the computed result for $\mu^{k *}$ could be negative. However, the Lagrangian multiplier theory guarantees that $\mu^{k *}$ should always be nonnegative since it is an inequality constraint 28b). This contradiction comes from the assumption that the constraint is active. It means that the constraint is inactive, therefore $\mu^{k *}$ should be zero. Based on this fact, we compute $\mu^{k *}$ by

$$
\mu^{k *}=\max \left\{0, \frac{\left(1+\frac{\rho}{\ell N}\right)\left\|\mathbf{S}_{\mathrm{F}} \mathbf{v}^{k}\right\|_{2}-\sqrt{\beta} \frac{\rho}{\ell N}\left\|\mathbf{S}_{\mathrm{D}} \mathbf{v}^{k}\right\|_{2}}{2\left(\beta\left\|\mathbf{S}_{\mathrm{F}} \mathbf{v}^{k}\right\|_{2}+\sqrt{\beta}\left\|\mathbf{S}_{\mathrm{D}} \mathbf{v}^{k}\right\|_{2}\right)}\right\} .
$$

\section{B. Solving the Subproblem 23b}

The problem $23 \mathrm{~b}$ can be equivalent to

$$
\begin{array}{ll}
\min _{\mathbf{x} \in \mathbb{C}^{\ell N}}\left\|\mathbf{x}-\mathbf{w}^{k}+\frac{\mathbf{y}_{2}^{k}}{\rho}\right\|_{2}^{2}, \\
\text { subject to } & \frac{\|\mathbf{x}\|_{\infty}^{2}}{\frac{1}{\ell N}\|\mathbf{x}\|_{2}^{2}}=\alpha .
\end{array}
$$

Similar to the ADMM-Direct algorithm, we also introduce auxiliary variables $t$ and $\mathbf{z}$ to express $\mathbf{x}$ by $\mathbf{x}=t \mathbf{z}$, where $t>0$ and $\|\mathbf{z}\|_{2}^{2}=1$. Plugging the auxiliary variables $t$ and $\mathbf{z}$ into the problem (34), we can obtain

$$
\begin{array}{ll}
\min _{\mathbf{z} \in \mathbb{C}^{\ell N}, t>0} & t^{2}-2 t \operatorname{Re}\left(\mathbf{z}^{H} \mathbf{b}^{k}\right), \\
\text { subject to } & \left|z_{i}\right|^{2} \leq \frac{\alpha}{\ell N}, \quad i=1, \cdots, \ell N, \\
& \|\mathbf{z}\|_{2}^{2}=1,
\end{array}
$$

where $\mathbf{b}^{k}=\mathbf{w}^{k}-\frac{\mathbf{y}_{2}^{k}}{\rho}$.

To solve $\mathbf{z}^{k+1}$, we can drop $t$ from the model (35) and formulate the following equivalent convex optimization model.

$$
\begin{array}{ll}
\max _{\mathbf{z} \in \mathbb{C}^{\ell N}} & \operatorname{Re}\left(\mathbf{z}^{H} \mathbf{b}^{k}\right), \\
\text { subject to } & \left|z_{i}\right|^{2} \leq \frac{\alpha}{\ell N}, \quad i=1, \cdots, \ell N, \\
& \|\mathbf{z}\|_{2}^{2} \leq 1 .
\end{array}
$$

By introducing the Lagrangian multiplier $\gamma^{k}>0$ for the constraint 36c), we can change the model (36) to

$$
\begin{array}{ll}
\min _{z_{i} \in \mathbb{C}, \gamma^{k}>0} & \sum_{i=1}^{\ell N}-\operatorname{Re}\left(z_{i}^{\dagger} b_{i}^{k}\right)+\gamma^{k}\left(\sum_{i=1}^{\ell N}\left|z_{i}\right|^{2}-1\right), \\
\text { subject to } & \left|z_{i}\right| \leq \sqrt{\frac{\alpha}{\ell N}}, \quad i=1, \cdots, \ell N .
\end{array}
$$

Since both the objective function (37a) and constraint 37b are treated separately in the variable $z_{i}$, solving the model 37 is equivalent to solving the following $\ell N$ subproblems, which 
can be performed in parallel.

$$
\begin{array}{ll}
\min _{z_{i}} \in \mathbb{C}, \gamma^{k}>0 & -\operatorname{Re}\left(z_{i}^{\dagger} b_{i}^{k}\right)+\gamma^{k}\left|z_{i}\right|^{2}, \\
\text { subject to } & \left|z_{i}\right| \leq \sqrt{\frac{\alpha}{\ell N}} .
\end{array}
$$

Moreover, since only one constraint is involved in (38), its optimal solution can be obtained through 39].

$$
z_{i}^{k+1}=\left\{\begin{array}{cl}
\frac{b_{i}^{k}}{2 \gamma^{k}}, & \frac{\left|b_{i}^{k}\right|}{2 \gamma^{k}}<\sqrt{\frac{\alpha}{\ell N}} \\
\sqrt{\frac{\alpha}{\ell N}} e^{j} \phi\left(b_{i}^{k}\right), & \text { otherwise }
\end{array}\right.
$$

where $\phi\left(b_{i}^{k}\right)$ represents the phase of $b_{i}^{k}$. Furthermore, since $\mathbf{z}^{k+1}$ should satisfy the constraint $\left\|\mathbf{z}^{k+1}\right\|_{2}^{2}=1$, the optimal Lagrangian multiplier $\gamma^{k *}$ can be determined by the binary section searching procedure as shown in Table I. After that, plugging the obtained $\mathbf{z}^{k+1}$ into the model 36 and simplifying it as a quadratic problem, we can get $t^{k+1}=$ $\operatorname{Re}\left(\mathbf{z}^{k+1 H} \mathbf{b}^{k}\right)$. At last, plugging $\mathbf{z}^{k+1}$ and $t^{k+1}$ into $\mathbf{x}=t \mathbf{z}$, we get the optimal solution $\mathrm{x}^{k+1}$ of (34).

\section{Solving the Subproblem (23c)}

Since the problem $23 \mathrm{c}$ is an unconstrained quadratic problem, its optimal solution $\left(\mathbf{u}^{k+1}, \mathbf{w}^{k+1}\right)$ should satisfy

$$
\begin{aligned}
& \nabla_{\mathbf{u}} L_{\rho}\left(\mathbf{x}^{k+1}, \mathbf{c}^{k+1}, \mathbf{u}^{k+1}, \mathbf{w}^{k+1}, \mathbf{y}_{1}^{k}, \mathbf{y}_{2}^{k}\right)=0, \\
& \nabla_{\mathbf{w}} L_{\rho}\left(\mathbf{x}^{k+1}, \mathbf{c}^{k+1}, \mathbf{u}^{k+1}, \mathbf{w}^{k+1}, \mathbf{y}_{1}^{k}, \mathbf{y}_{2}^{k}\right)=0 .
\end{aligned}
$$

That is $\left(\mathbf{u}^{k+1}, \mathbf{w}^{k+1}\right)$ is the solution of

$$
\begin{aligned}
& -\mathbf{y}_{1}^{k}+\tilde{\rho}\left(\mathbf{u}^{k+1}-\mathbf{w}^{k+1}\right)-\rho\left(\mathbf{A} \mathbf{c}^{k+1}-\mathbf{u}^{k+1}\right)=0, \\
& -\mathbf{y}_{2}^{k}-\tilde{\rho}\left(\mathbf{u}^{k+1}-\mathbf{w}^{k+1}\right)-\rho\left(\mathbf{x}^{k+1}-\mathbf{w}^{k+1}\right)=0 .
\end{aligned}
$$

Solving these two equations, we can get

$$
\begin{aligned}
\mathbf{u}^{k+1} & =\frac{\mathbf{y}_{1}^{k}+\tilde{\rho} \mathbf{x}^{k+1}+(\rho+\tilde{\rho}) \operatorname{IFFT}_{\ell}\left(\mathbf{c}^{k+1}\right)}{2 \tilde{\rho}+\rho}, \\
\mathbf{w}^{k+1} & =\frac{\mathbf{y}_{2}^{k}+(\tilde{\rho}+\rho) \mathbf{x}^{k+1}+\tilde{\rho} \operatorname{IFFT}_{\ell}\left(\mathbf{c}^{k+1}\right)}{2 \tilde{\rho}+\rho} .
\end{aligned}
$$

\section{APPENDIX D}

\section{ProOF OF THEOREM 2}

First, we can see that the ADMM-Relax algorithm 23) can guarantee the resulting $\mathbf{c}^{k+1}$ and $\mathbf{x}^{k+1}$ to satisfy $21 \mathrm{~b}$ and (21c) respectively. Thus, without loss of generality we assume that $\mathrm{c}^{1}$ and $\mathrm{x}^{1}$ satisfy (21b) and (21c) respectively.

Since $\mathbf{c}^{k+1}$ and $\mathbf{x}^{k+1}$ are the minimizers of the problems (23a) and 23b respectively, we have

$$
\begin{array}{r}
L_{\rho}\left(\mathbf{c}^{k}, \mathbf{x}^{k}, \mathbf{u}^{k}, \mathbf{w}^{k}, \mathbf{y}_{1}^{k}, \mathbf{y}_{2}^{k}\right) \\
-L_{\rho}\left(\mathbf{c}^{k+1}, \mathbf{x}^{k}, \mathbf{u}^{k}, \mathbf{w}^{k}, \mathbf{y}_{1}^{k}, \mathbf{y}_{2}^{k}\right) \geq 0, \\
L_{\rho}\left(\mathbf{c}^{k+1}, \mathbf{x}^{k}, \mathbf{u}^{k}, \mathbf{w}^{k}, \mathbf{y}_{1}^{k}, \mathbf{y}_{2}^{k}\right) \\
-L_{\rho}\left(\mathbf{c}^{k+1}, \mathbf{x}^{k+1}, \mathbf{u}^{k}, \mathbf{w}^{k}, \mathbf{y}_{1}^{k}, \mathbf{y}_{2}^{k}\right) \geq 0 .
\end{array}
$$

Moreover, based on the Taylor expansion, we can obtain

$$
\begin{aligned}
& L_{\rho}\left(\mathbf{c}^{k+1}, \mathbf{x}^{k+1}, \mathbf{u}^{k}, \mathbf{w}^{k}, \mathbf{y}_{1}^{k}, \mathbf{y}_{2}^{k}\right) \\
& \quad-L_{\rho}\left(\mathbf{c}^{k+1}, \mathbf{x}^{k+1}, \mathbf{u}^{k+1}, \mathbf{w}^{k+1}, \mathbf{y}_{1}^{k}, \mathbf{y}_{2}^{k}\right) \\
= & \frac{1}{2}\left[\begin{array}{c}
\mathbf{u}^{k}-\mathbf{u}^{k+1} \\
\mathbf{w}^{k}-\mathbf{w}^{k+1}
\end{array}\right]{ }^{H}\left[\begin{array}{cc}
\tilde{\rho}+\rho & -\tilde{\rho} \\
-\tilde{\rho} & \tilde{\rho}+\rho
\end{array}\right]\left[\begin{array}{c}
\mathbf{u}^{k}-\mathbf{u}^{k+1} \\
\mathbf{w}^{k}-\mathbf{w}^{k+1}
\end{array}\right],
\end{aligned}
$$

where the linear term is dropped since $\mathbf{u}^{k+1}$ and $\mathbf{w}^{k+1}$ are the minimizers of the problem $23 \mathrm{c}$, that is

$$
\begin{gathered}
\nabla_{\mathbf{u}} L_{\rho}\left(\mathbf{x}^{k+1}, \mathbf{c}^{k+1}, \mathbf{u}^{k+1}, \mathbf{w}^{k+1}, \mathbf{y}_{1}^{k}, \mathbf{y}_{2}^{k}\right)=0, \\
\nabla_{\mathbf{w}} L_{\rho}\left(\mathbf{x}^{k+1}, \mathbf{c}^{k+1}, \mathbf{u}^{k+1}, \mathbf{w}^{k+1}, \mathbf{y}_{1}^{k}, \mathbf{y}_{2}^{k}\right)=0 .
\end{gathered}
$$

Furthermore, according to 23d and 23e , we have

$$
\begin{aligned}
& L_{\rho}\left(\mathbf{c}^{k+1}, \mathbf{x}^{k+1}, \mathbf{u}^{k+1}, \mathbf{w}^{k+1}, \mathbf{y}_{1}^{k}, \mathbf{y}_{2}^{k}\right) \\
& \quad-L_{\rho}\left(\mathbf{c}^{k+1}, \mathbf{x}^{k+1}, \mathbf{u}^{k+1}, \mathbf{w}^{k+1}, \mathbf{y}_{1}^{k+1}, \mathbf{y}_{2}^{k}\right) \\
= & -\frac{1}{\rho}\left\|\mathbf{y}_{1}^{k+1}-\mathbf{y}_{1}^{k}\right\|_{2}^{2},
\end{aligned}
$$

and

$$
\begin{aligned}
& L_{\rho}\left(\mathbf{c}^{k+1}, \mathbf{x}^{k+1}, \mathbf{u}^{k+1}, \mathbf{w}^{k+1}, \mathbf{y}_{1}^{k+1}, \mathbf{y}_{2}^{k}\right) \\
& \quad-L_{\rho}\left(\mathbf{c}^{k+1}, \mathbf{x}^{k+1}, \mathbf{u}^{k+1}, \mathbf{w}^{k+1}, \mathbf{y}_{1}^{k+1}, \mathbf{y}_{2}^{k+1}\right) \\
= & -\frac{1}{\rho}\left\|\mathbf{y}_{2}^{k+1}-\mathbf{y}_{2}^{k}\right\|_{2}^{2} .
\end{aligned}
$$

Adding both sides of (43)-477, we can obtain

$$
\begin{aligned}
& L_{\rho}\left(\mathbf{c}^{k}, \mathbf{x}^{k}, \mathbf{u}^{k}, \mathbf{w}^{k}, \mathbf{y}_{1}^{k}, \mathbf{y}_{2}^{k}\right) \\
& -L_{\rho}\left(\mathbf{c}^{k+1}, \mathbf{x}^{k+1}, \mathbf{u}^{k+1}, \mathbf{w}^{k+1}, \mathbf{y}_{1}^{k+1}, \mathbf{y}_{2}^{k+1}\right) \\
& \geq \frac{1}{2}\left[\begin{array}{c}
\mathbf{u}^{k}-\mathbf{u}^{k+1} \\
\mathbf{w}^{k}-\mathbf{w}^{k+1}
\end{array}\right]^{H}\left[\begin{array}{cc}
\tilde{\rho}+\rho & -\tilde{\rho} \\
-\tilde{\rho} & \tilde{\rho}+\rho
\end{array}\right]\left[\begin{array}{c}
\mathbf{u}^{k}-\mathbf{u}^{k+1} \\
\mathbf{w}^{k}-\mathbf{w}^{k+1}
\end{array}\right] \\
& -\frac{1}{\rho}\left(\left\|\mathbf{y}_{1}^{k+1}-\mathbf{y}_{1}^{k}\right\|_{2}^{2}+\left\|\mathbf{y}_{2}^{k+1}-\mathbf{y}_{2}^{k}\right\|_{2}^{2}\right) \text {. }
\end{aligned}
$$

Setting the gradient of the objective function in 23c with respect to $\mathbf{u}$ as zero, we have the following derivations

$$
\begin{aligned}
0 & =\nabla_{\mathbf{u}} L_{\rho}\left(\mathbf{c}^{k+1}, \mathbf{x}^{k+1}, \mathbf{u}^{k+1}, \mathbf{w}^{k+1}, \mathbf{y}_{1}^{k}, \mathbf{y}_{2}^{k}\right) \\
& =-\mathbf{y}_{1}^{k}+\tilde{\rho}\left(\mathbf{u}^{k+1}-\mathbf{w}^{k+1}\right)-\rho\left(\mathbf{A c}^{k+1}-\mathbf{u}^{k+1}\right) \\
& =-\mathbf{y}_{1}^{k}+\tilde{\rho}\left(\mathbf{u}^{k+1}-\mathbf{w}^{k+1}\right)+\left(\mathbf{y}_{1}^{k}-\mathbf{y}_{1}^{k+1}\right) \\
& =\tilde{\rho}\left(\mathbf{u}^{k+1}-\mathbf{w}^{k+1}\right)-\mathbf{y}_{1}^{k+1},
\end{aligned}
$$

then we can get

$$
\mathbf{y}_{1}^{k+1}=\tilde{\rho}\left(\mathbf{u}^{k+1}-\mathbf{w}^{k+1}\right),
$$

where the third equality comes from (23d). So, we have

$$
\begin{aligned}
& \left\|\mathbf{y}_{1}^{k+1}-\mathbf{y}_{1}^{k}\right\|_{2}^{2}=\left\|\tilde{\rho}\left(\mathbf{u}^{k+1}-\mathbf{w}^{k+1}\right)-\tilde{\rho}\left(\mathbf{u}^{k}-\mathbf{w}^{k}\right)\right\|_{2}^{2} \\
& =\tilde{\rho}^{2}\left\|\left(\mathbf{u}^{k}-\mathbf{u}^{k+1}\right)-\left(\mathbf{w}^{k}-\mathbf{w}^{k+1}\right)\right\|_{2}^{2} \\
& =\left[\begin{array}{c}
\mathbf{u}^{k}-\mathbf{u}^{k+1} \\
\mathbf{w}^{k}-\mathbf{w}^{k+1}
\end{array}\right]^{H}\left[\begin{array}{cc}
\tilde{\rho}^{2} & -\tilde{\rho}^{2} \\
-\tilde{\rho}^{2} & \tilde{\rho}^{2}
\end{array}\right]\left[\begin{array}{c}
\mathbf{u}^{k}-\mathbf{u}^{k+1} \\
\mathbf{w}^{k}-\mathbf{w}^{k+1}
\end{array}\right] .
\end{aligned}
$$

Through similar derivations for the gradient of the problem (23c) with respect to $w$, we can obtain (51) and (52).

$$
\begin{gathered}
\mathbf{y}_{2}^{k+1}=-\tilde{\rho}\left(\mathbf{u}^{k+1}-\mathbf{w}^{k+1}\right), \\
\left\|\mathbf{y}_{2}^{k+1}-\mathbf{y}_{2}^{k}\right\|_{2}^{2}=\left\|\tilde{\rho}\left(\mathbf{u}^{k}-\mathbf{w}^{k}\right)-\tilde{\rho}\left(\mathbf{u}^{k+1}-\mathbf{w}^{k+1}\right)\right\|_{2}^{2} \\
=\left[\begin{array}{c}
\mathbf{u}^{k}-\mathbf{u}^{k+1} \\
\mathbf{w}^{k}-\mathbf{w}^{k+1}
\end{array}\right]^{H}\left[\begin{array}{cc}
\tilde{\rho}^{2} & -\tilde{\rho}^{2} \\
-\tilde{\rho}^{2} & \tilde{\rho}^{2}
\end{array}\right]\left[\begin{array}{c}
\mathbf{u}^{k}-\mathbf{u}^{k+1} \\
\mathbf{w}^{k}-\mathbf{w}^{k+1}
\end{array}\right] .
\end{gathered}
$$


Plugging (50) and (52) into (48), we obtain

$$
\begin{aligned}
& L_{\rho}\left(\mathbf{c}^{k}, \mathbf{x}^{k}, \mathbf{u}^{k}, \mathbf{w}^{k}, \mathbf{y}_{1}^{k}, \mathbf{y}_{2}^{k}\right) \\
& \quad-L_{\rho}\left(\mathbf{c}^{k+1}, \mathbf{x}^{k+1}, \mathbf{u}^{k+1}, \mathbf{w}^{k+1}, \mathbf{y}_{1}^{k+1}, \mathbf{y}_{2}^{k+1}\right) \\
\geq & {\left[\begin{array}{c}
\mathbf{u}^{k}-\mathbf{u}^{k+1} \\
\mathbf{w}^{k}-\mathbf{w}^{k+1}
\end{array}\right]^{H} \boldsymbol{Q}\left[\begin{array}{c}
\mathbf{u}^{k}-\mathbf{u}^{k+1} \\
\mathbf{w}^{k}-\mathbf{w}^{k+1}
\end{array}\right], }
\end{aligned}
$$

where $\boldsymbol{Q}=\left[\begin{array}{lr}\frac{\tilde{\rho}+\rho}{2}-\frac{2 \tilde{\rho}^{2}}{\rho} & \frac{2 \tilde{\rho}^{2}}{\rho}-\frac{\tilde{\rho}}{2} \\ \frac{2 \tilde{\rho}^{2}}{\rho}-\frac{\tilde{\rho}}{2} & \frac{\tilde{\rho}+\rho}{2}-\frac{2 \tilde{\rho}^{2}}{\rho}\end{array}\right]$ and its eigenvalues $\lambda(\mathbf{Q})$ are $\frac{\rho}{2}$ and $\frac{\rho^{2}+2 \rho \tilde{\rho}-8 \tilde{\rho}^{2}}{2 \rho}$ respectively. We can verify that, when $\rho>2 \tilde{\rho}>0$, the matrix $\mathbf{Q}$ is positive definite. Then, (53) can be simplified as

$$
\begin{aligned}
& \quad L_{\rho}\left(\mathbf{c}^{k}, \mathbf{x}^{k}, \mathbf{u}^{k}, \mathbf{w}^{k}, \mathbf{y}_{1}^{k}, \mathbf{y}_{2}^{k}\right) \\
& \quad-L_{\rho}\left(\mathbf{c}^{k+1}, \mathbf{x}^{k+1}, \mathbf{u}^{k+1}, \mathbf{w}^{k+1}, \mathbf{y}_{1}^{k+1}, \mathbf{y}_{2}^{k+1}\right) \\
& \geq \lambda_{\min }(\mathbf{Q})\left(\left\|\mathbf{u}^{k+1}-\mathbf{u}^{k}\right\|_{2}^{2}+\left\|\mathbf{w}^{k+1}-\mathbf{w}^{k}\right\|_{2}^{2}\right) .
\end{aligned}
$$

Adding both sides of the above inequality from $k=1,2, \ldots$, we can get

$$
\begin{aligned}
& L_{\rho}\left(\mathbf{c}^{1}, \mathbf{x}^{1}, \mathbf{u}^{1}, \mathbf{w}^{1}, \mathbf{y}_{1}^{1}, \mathbf{y}_{2}^{1}\right) \\
& \quad-\lim _{k \rightarrow+\infty} L_{\rho}\left(\mathbf{c}^{k+1}, \mathbf{x}^{k+1}, \mathbf{u}^{k+1}, \mathbf{w}^{k+1}, \mathbf{y}_{1}^{k+1}, \mathbf{y}_{2}^{k+1}\right) \\
& \geq \lambda_{\min }(\mathbf{Q})\left(\sum_{k=1}^{+\infty}\left\|\mathbf{u}^{k+1}-\mathbf{u}^{k}\right\|_{2}^{2}+\sum_{k=1}^{+\infty}\left\|\mathbf{w}^{k+1}-\mathbf{w}^{k}\right\|_{2}^{2}\right)>0 .
\end{aligned}
$$

Moreover, plugging (49) and 51) into the augmented Lagrangian function $L_{\rho}\left(\mathbf{c}^{k+1}, \mathbf{x}^{k+1}, \mathbf{u}^{k+1}, \mathbf{w}^{k+1}, \mathbf{y}_{1}^{k+1}, \mathbf{y}_{2}^{k+1}\right)$, we can derived it as

$$
\begin{aligned}
& L_{\rho}\left(\mathbf{c}^{k+1}, \mathbf{x}^{k+1}, \mathbf{u}^{k+1}, \mathbf{w}^{k+1}, \mathbf{y}_{1}^{k+1}, \mathbf{y}_{2}^{k+1}\right) \\
= & \frac{1}{2}\left\|\mathbf{S}_{\mathrm{D}}\left(\mathbf{c}^{k+1}-\mathbf{c}_{\mathrm{o}}\right)\right\|_{2}^{2}+\tilde{\rho}\left\|\mathbf{A} \mathbf{c}^{k+1}-\frac{1}{2} \mathbf{u}^{k+1}-\frac{1}{2} \mathbf{w}^{k+1}\right\|_{2}^{2} \\
+ & \left(\frac{\rho}{2}-\tilde{\rho}\right)\left(\left\|\mathbf{A} \mathbf{c}^{k+1}-\mathbf{u}^{k+1}\right\|_{2}^{2}+\left\|\mathbf{x}^{k+1}-\mathbf{w}^{k+1}\right\|_{2}^{2}\right) \\
+ & \tilde{\rho}\left\|\mathbf{x}^{k+1}-\frac{1}{2} \mathbf{u}^{k+1}-\frac{1}{2} \mathbf{w}^{k+1}\right\|_{2}^{2} .
\end{aligned}
$$

Since $\rho>2 \tilde{\rho}$, we see that

$$
L_{\rho}\left(\mathbf{c}^{k+1}, \mathbf{x}^{k+1}, \mathbf{u}^{k+1}, \mathbf{w}^{k+1}, \mathbf{y}_{1}^{k+1}, \mathbf{y}_{2}^{k+1}\right) \geq 0, \quad \forall k .
$$

We can conclude from (55) and (57) that

$$
\begin{aligned}
& \lim _{k \rightarrow+\infty} \mathbf{u}^{k+1}-\mathbf{u}^{k}=0, \\
& \lim _{k \rightarrow+\infty} \mathbf{w}^{k+1}-\mathbf{w}^{k}=0 .
\end{aligned}
$$

Plugging (58) into (50) and (52) respectively, we get

$$
\begin{aligned}
& \lim _{k \rightarrow+\infty} \mathbf{y}_{1}^{k+1}-\mathbf{y}_{1}^{k}=0, \\
& \lim _{k \rightarrow+\infty} \mathbf{y}_{2}^{k+1}-\mathbf{y}_{2}^{k}=0 .
\end{aligned}
$$

Combining the above results with (23d and $(23 \mathrm{e}$, we derive the following equalities

$$
\begin{aligned}
& \lim _{k \rightarrow+\infty} \mathbf{A} \mathbf{c}^{k+1}-\mathbf{u}^{k+1}=0, \\
& \lim _{k \rightarrow+\infty} \mathbf{x}^{k+1}-\mathbf{w}^{k+1}=0 .
\end{aligned}
$$

Next, let us show that $\mathbf{c}^{k+1}, \mathbf{x}^{k+1}, \mathbf{u}^{k+1}, \mathbf{w}^{k+1}, \mathbf{y}_{1}^{k+1}$ and $\mathbf{y}_{2}^{k+1}$ are bounded as $k \rightarrow+\infty$.
Plugging the limitation results 58 and $(60)$ into 56 , we can derive $L_{\rho}\left(\mathbf{c}^{k+1}, \mathbf{x}^{k+1}, \mathbf{u}^{k+1}, \mathbf{w}^{k+1}, \mathbf{y}_{1}^{k+1}, \mathbf{y}_{2}^{k+1}\right)$ as

$$
\begin{aligned}
& \lim _{k \rightarrow+\infty} L_{\rho}\left(\mathbf{c}^{k+1}, \mathbf{x}^{k+1}, \mathbf{u}^{k+1}, \mathbf{w}^{k+1}, \mathbf{y}_{1}^{k+1}, \mathbf{y}_{2}^{k+1}\right) \\
= & \lim _{k \rightarrow+\infty} \frac{1}{2}\left\|\mathbf{S}_{\mathrm{D}}\left(\mathbf{c}^{k+1}-\mathbf{c}_{\mathrm{o}}\right)\right\|_{2}^{2}+\lim _{k \rightarrow+\infty} \frac{\tilde{\rho}}{2}\left\|\mathbf{u}^{k+1}-\mathbf{w}^{k+1}\right\|_{2}^{2} \\
\leq & L_{\rho}\left(\mathbf{c}^{1}, \mathbf{x}^{1}, \mathbf{u}^{1}, \mathbf{w}^{1}, \mathbf{y}_{1}^{1}, \mathbf{y}_{2}^{1}\right),
\end{aligned}
$$

which means that $\left\|\mathbf{S}_{\mathrm{D}} \mathbf{c}^{k+1}\right\|_{2}^{2}$ and $\left\|\mathbf{u}^{k+1}-\mathbf{w}^{k+1}\right\|_{2}^{2}$ are bounded as $k \rightarrow+\infty$. Moreover, since $\mathbf{c}^{k+1}$ satisfies the constraint $3 \mathrm{c}$, we can conclude that $\left\|\mathbf{S}_{\mathrm{F}} \mathbf{c}^{k+1}\right\|_{2}^{2}$ is also bounded. Since $\mathbf{S}_{\mathrm{D}}+\mathbf{S}_{\mathrm{F}}$ is an identity matrix, we get that $\left\|\mathbf{c}^{k+1}\right\|_{2}$ is bounded as $k \rightarrow+\infty$. Plugging this result into 60a), we can see that $\left\|\mathbf{u}^{k+1}\right\|_{2}$ is bounded as $k \rightarrow+\infty$, which leads to $\left\|\mathbf{w}^{k+1}\right\|_{2}$ is also bounded. So, we can get that $\left\|\mathrm{x}^{k+1}\right\|_{2}$ is bounded from 60b). Furthermore, since $\mathbf{y}_{1}^{k+1}=\tilde{\rho}\left(\mathbf{u}^{k+1}-\mathbf{w}^{k+1}\right)$ and $\mathbf{y}_{2}^{k+1}=-\tilde{\rho}\left(\mathbf{u}^{k+1}-\mathbf{w}^{k+1}\right)$, we can conclude that the Lagrangian multipliers $\mathbf{y}_{1}^{k+1}$ and $\mathbf{y}_{2}^{k+1}$ are also bounded.

Combining the above bounded results with (58) and (59), we can obtain the following results

$$
\begin{array}{ll}
\lim _{k \rightarrow+\infty} \mathbf{u}^{k}=\mathbf{u}^{*}, & \lim _{k \rightarrow+\infty} \mathbf{w}^{k}=\mathbf{w}^{*}, \\
\lim _{k \rightarrow+\infty} \mathbf{y}_{1}^{k}=\mathbf{y}_{1}^{*}, & \lim _{k \rightarrow+\infty} \mathbf{y}_{2}^{k}=\mathbf{y}_{2}^{*}, \quad \mathbf{y}_{1}^{*}=-\mathbf{y}_{2}^{*} .
\end{array}
$$

Since $\mathbf{u}^{k}$ and $\mathbf{w}^{k}$ are convergent as $k \rightarrow+\infty$, we have $\lim _{k \rightarrow+\infty} \mathbf{A} \mathbf{c}^{k}=\mathbf{u}^{*}$ and $\lim _{k \rightarrow+\infty} \mathbf{x}^{k}=\mathbf{w}^{*}$. Moreover, since $\mathbf{A}$ is full rank in columns, it means that $\lim _{k \rightarrow+\infty} \mathbf{c}^{k}=\ell N \mathbf{A}^{H} \mathbf{u}^{*}$. We can further obtain

$$
\begin{array}{cc}
\lim _{k \rightarrow+\infty} \mathbf{c}^{k}=\mathbf{c}^{*}, & \lim _{k \rightarrow+\infty} \mathbf{x}^{k}=\mathbf{x}^{*}, \\
\mathbf{A} \mathbf{c}^{*}=\mathbf{u}^{*}, & \mathbf{x}^{*}=\mathbf{w}^{*},
\end{array}
$$

which concludes the proof of the first part of Theorem 2 .

Next, we consider to prove the second part of Theorem 2 that $\left(\mathbf{c}^{*}, \mathbf{x}^{*}, \mathbf{u}^{*}, \mathbf{w}^{*}\right)$ is a KKT point of the model 21). Its proof is similar to the proof presented in Appendix B. Here, we denote $\tilde{L}\left(\mathbf{c}, \mathbf{x}, \mathbf{u}, \mathbf{w}, \mathbf{y}_{1}, \mathbf{y}_{2}, \mu, \gamma\right), \tilde{L}^{\mathbf{c}}\left(\mathbf{c}, \mathbf{y}_{1}, \mu\right), \tilde{L}^{\mathbf{x}}\left(\mathbf{x}, \mathbf{y}_{2}, \gamma\right)$, $\tilde{L}^{\mathbf{u}}\left(\mathbf{u}, \mathbf{w}, \mathbf{y}_{1}\right)$ and $\tilde{L}^{\mathbf{w}}\left(\mathbf{u}, \mathbf{w}, \mathbf{y}_{2}\right)$ as the Lagrangian functions of the problems 21, 23a, 23b , and 23c) with respect to $\mathbf{u}$ and $\mathbf{w}$ respectively. $\mu$ and $\gamma$ are the Lagrangian multipliers corresponding to the constraints (21c) and 21b respectively. When ADMM-Relax algorithm is convergent, we let $\mu^{*}$ and $\gamma^{*}$ denote the corresponding optimal Lagrangian multipliers. Since in every ADMM-Relax iteration $\mathbf{c}^{k+1}$ and $\mathbf{x}^{k+1}$ are always located in the feasible region, we can see that $\mathbf{c}^{*}$ and $\mathrm{x}^{*}$ satisfy the feasibility conditions, i.e.,

$$
\mathbf{c}^{*} \in \mathcal{C}, \quad \mathbf{x}^{*} \in \mathcal{X} .
$$

Since $\mu^{k *} \geq 0$ in every ADMM-Relax iteration, it means

$$
\mu^{*} \geq 0 \text {. }
$$

Moreover, from (33), we see that the value of $\mu^{k *}, \forall k$, is nonzero or zero corresponding to the constraint $(28 \mathrm{~b})$, which is active or inactive respectively. It means that $\mu^{*}$ satisfies the complementary slackness condition, i.e.,

$$
\mu^{*}\left(\left\|\mathbf{S}_{\mathrm{F}} \mathbf{c}^{*}\right\|_{2}^{2}-\beta\left\|\mathbf{S}_{\mathrm{D}} \mathbf{c}^{*}\right\|_{2}^{2}\right)=0 .
$$


Furthermore, since $\mathbf{c}^{k+1}, \mathbf{x}^{k+1}, \mathbf{u}^{k+1}$, and $\mathbf{w}^{k+1}$ are the minimizers of the problems 23a , 23b), and 23c respectively in the $k$ th ADMM-Relax iteration, so they should satisfy

$$
\begin{aligned}
& \nabla_{\mathbf{c}} \tilde{L}^{\mathbf{c}}\left(\mathbf{c}^{k+1}, \mathbf{y}_{1}^{k}, \mu^{k+1}\right)+\rho \mathbf{A}^{H}\left(\mathbf{A} \mathbf{c}^{k+1}-\mathbf{u}^{k}\right)=0, \\
& \nabla_{\mathbf{x}} \tilde{L}^{\mathbf{x}}\left(\mathbf{x}^{k+1}, \mathbf{y}_{2}^{k}, \gamma^{k+1}\right)+\rho\left(\mathbf{x}^{k+1}-\mathbf{w}^{k}\right)=0, \\
& \nabla_{\mathbf{u}} \tilde{L}^{\mathbf{u}}\left(\mathbf{u}^{k+1}, \mathbf{w}^{k+1}, \mathbf{y}_{1}^{k}\right)-\rho\left(\mathbf{A} \mathbf{c}^{k+1}-\mathbf{u}^{k+1}\right)=0, \\
& \nabla_{\mathbf{w}} \tilde{L}^{\mathbf{w}}\left(\mathbf{u}^{k+1}, \mathbf{w}^{k+1}, \mathbf{y}_{2}^{k}\right)-\rho\left(\mathbf{x}^{k+1}-\mathbf{w}^{k+1}\right)=0 .
\end{aligned}
$$

According to the convergence results 62 and 63, we can change 67) to 68) when $k \rightarrow+\infty$.

$$
\begin{array}{ll}
\nabla_{\mathbf{c}} \tilde{L}^{\mathbf{c}}\left(\mathbf{c}^{*}, \mathbf{y}_{1}^{*}, \mu^{*}\right)=0, & \nabla_{\mathbf{x}} \tilde{L}^{\mathbf{x}}\left(\mathbf{x}^{*}, \mathbf{y}_{2}^{*}, \gamma^{*}\right)=0, \\
\nabla_{\mathbf{u}} \tilde{L}^{\mathbf{u}}\left(\mathbf{u}^{*}, \mathbf{w}^{*} \mathbf{y}_{1}^{*}\right)=0, & \nabla_{\mathbf{w}} \tilde{L}^{\mathbf{w}}\left(\mathbf{u}^{*}, \mathbf{w}^{*}, \mathbf{y}_{2}^{*}\right)=0 .
\end{array}
$$

Since there are

$$
\begin{aligned}
& \nabla_{\mathbf{c}} \tilde{L}^{\mathbf{c}}\left(\mathbf{c}^{*}, \mathbf{y}_{1}^{*}, \mu^{*}\right)=\nabla_{\mathbf{c}} \tilde{L}\left(\mathbf{c}^{*}, \mathbf{x}^{*}, \mathbf{u}^{*}, \mathbf{w}^{*}, \mathbf{y}_{1}^{*}, \mathbf{y}_{2}^{*}\right), \\
& \nabla_{\mathbf{x}} \tilde{L}^{\mathbf{x}}\left(\mathbf{x}^{*}, \mathbf{y}_{2}^{*}, \gamma^{*}\right)=\nabla_{\mathbf{x}} \tilde{L}\left(\mathbf{c}^{*}, \mathbf{x}^{*}, \mathbf{u}^{*}, \mathbf{w}^{*}, \mathbf{y}_{1}^{*}, \mathbf{y}_{2}^{*}\right), \\
& \nabla_{\mathbf{u}} \tilde{L}^{\mathbf{u}}\left(\mathbf{u}^{*}, \mathbf{w}^{*} \mathbf{y}_{1}^{*}\right)=\nabla_{\mathbf{u}} \tilde{L}\left(\mathbf{c}^{*}, \mathbf{x}^{*}, \mathbf{u}^{*}, \mathbf{w}^{*}, \mathbf{y}_{1}^{*}, \mathbf{y}_{2}^{*}\right), \\
& \nabla_{\mathbf{w}} \tilde{L}^{\mathbf{w}}\left(\mathbf{u}^{*}, \mathbf{w}^{*}, \mathbf{y}_{2}^{*}\right)=\nabla_{\mathbf{w}} \tilde{L}\left(\mathbf{c}^{*}, \mathbf{x}^{*}, \mathbf{u}^{*}, \mathbf{w}^{*}, \mathbf{y}_{1}^{*}, \mathbf{y}_{2}^{*}\right),
\end{aligned}
$$

$\left(\mathbf{c}^{*}, \mathbf{x}^{*}, \mathbf{u}^{*}, \mathbf{w}^{*}\right)$ should also satisfy

$$
\begin{aligned}
& \nabla_{\mathbf{c}} \tilde{L}\left(\mathbf{c}^{*}, \mathbf{x}^{*}, \mathbf{u}^{*}, \mathbf{w}^{*}, \mathbf{y}_{1}^{*}, \mathbf{y}_{2}^{*}\right)=0, \\
& \nabla_{\mathbf{x}} \tilde{L}\left(\mathbf{c}^{*}, \mathbf{x}^{*}, \mathbf{u}^{*}, \mathbf{w}^{*}, \mathbf{y}_{1}^{*}, \mathbf{y}_{2}^{*}\right)=0, \\
& \nabla_{\mathbf{u}} \tilde{L}\left(\mathbf{c}^{*}, \mathbf{x}^{*}, \mathbf{u}^{*}, \mathbf{w}^{*}, \mathbf{y}_{1}^{*}, \mathbf{y}_{2}^{*}\right)=0, \\
& \nabla_{\mathbf{w}} \tilde{L}\left(\mathbf{c}^{*}, \mathbf{x}^{*}, \mathbf{u}^{*}, \mathbf{w}^{*}, \mathbf{y}_{1}^{*}, \mathbf{y}_{2}^{*}\right)=0 .
\end{aligned}
$$

Combining 64, 65, 66, and 69, we can conclude that $\left(\mathbf{c}^{*}, \mathbf{x}^{*}, \mathbf{u}^{*}, \mathbf{w}^{*}\right)$ is some KKT point of the model (21).

To prove the third part of Theorem 2, we need to prove that $\left(\mathbf{c}^{*}, \mathbf{x}^{*}\right)$, combining the Lagrangian multipliers $\mathbf{y}^{*}, \mu^{*}$ and $\gamma^{*}$ satisfies the following KKT conditions

$$
\begin{aligned}
& \mathbf{c}^{*} \in \mathcal{C}, \quad \mathbf{x}^{*} \in \mathcal{X}, \\
& \lim _{\tilde{\rho} \rightarrow+\infty}\left\|\mathbf{A} \mathbf{c}^{*}-\mathbf{x}^{*}\right\|_{2}^{2}=0, \\
& \mu^{*} \geq 0, \\
& \mu^{*}\left(\left\|\mathbf{S}_{\mathrm{F}} \mathbf{c}^{*}\right\|_{2}^{2}-\beta\left\|\mathbf{S}_{\mathrm{D}} \mathbf{c}^{*}\right\|_{2}^{2}\right)=0, \\
& \nabla_{\mathbf{c}} L\left(\mathbf{c}^{*}, \mathbf{x}^{*}, \gamma^{*}, \mu^{*}, \mathbf{y}^{*}\right)=0, \\
& \nabla_{\mathbf{x}} L\left(\mathbf{c}^{*}, \mathbf{x}^{*}, \gamma^{*}, \mu^{*}, \mathbf{y}^{*}\right)=0,
\end{aligned}
$$

where $\gamma, \mu$ and $\mathbf{y}$ are the Lagrangian multipliers corresponding to the constraints (3b), 3c), and (3d) respectively, and $L(\mathbf{c}, \mathbf{x}, \gamma, \mu, \mathbf{y})$ is the Lagrangian function of the model (3). Notice here $\mathbf{y}^{*}=\mathbf{y}_{1}^{*}=-\mathbf{y}_{2}^{*}$. The proof for $\left(\mathbf{c}^{*}, \mathbf{x}^{*}, \gamma^{*}, \mu^{*}, \mathbf{y}^{*}\right)$ satisfying (70a), 170c), (70d) (70e), and (70f) are the same as 64, 65) and 66.

Here, we only need to prove that $\left(\mathbf{c}^{*}, \mathbf{x}^{*}, \gamma^{*}, \mu^{*}, \mathbf{y}^{*}\right)$ also satisfies (70b). According to 61, we have

$$
\begin{aligned}
& L_{\rho}\left(\mathbf{c}^{*}, \mathbf{x}^{*}, \mathbf{u}^{*}, \mathbf{w}^{*}, \mathbf{y}_{1}^{*}, \mathbf{y}_{2}^{*}\right) \\
= & \frac{1}{2}\left\|\mathbf{S}_{\mathrm{D}}\left(\mathbf{c}^{*}-\mathbf{c}_{\mathrm{o}}\right)\right\|_{2}^{2}+\frac{\tilde{\rho}}{2}\left\|\mathbf{u}^{*}-\mathbf{w}^{*}\right\|_{2}^{2} \\
\leq & L_{\rho}\left(\mathbf{c}^{1}, \mathbf{x}^{1}, \mathbf{u}^{1}, \mathbf{w}^{1}, \mathbf{y}_{1}^{1}, \mathbf{y}_{2}^{1}\right) .
\end{aligned}
$$

If $\mathbf{c}^{1} \in \mathcal{C}, \mathbf{x}^{1} \in \mathcal{X}, \mathbf{A} \mathbf{c}^{1}=\mathbf{x}^{1}$ and $\mathbf{u}^{1}=\mathbf{w}^{1}$, then

$$
L_{\rho}\left(\mathbf{c}^{1}, \mathbf{x}^{1}, \mathbf{u}^{1}, \mathbf{w}^{1}, \mathbf{y}_{1}^{1}, \mathbf{y}_{2}^{1}\right)=\frac{1}{2}\left\|\mathbf{S}_{\mathrm{D}}\left(\mathbf{c}^{1}-\mathbf{c}_{\mathrm{o}}\right)\right\|_{2}^{2} \text {. }
$$

Then, we can change (71) to

$$
\left\|\mathbf{u}^{*}-\mathbf{w}^{*}\right\|_{2}^{2} \leq \frac{1}{\tilde{\rho}}\left(\left\|\mathbf{S}_{\mathrm{D}}\left(\mathbf{c}^{1}-\mathbf{c}_{\mathrm{o}}\right)\right\|_{2}^{2}-\left\|\mathbf{S}_{\mathrm{D}}\left(\mathbf{c}^{*}-\mathbf{c}_{\mathrm{o}}\right)\right\|_{2}^{2}\right) \text {. }
$$

Moreover, since $\mathbf{A} \mathbf{c}^{*}=\mathbf{u}^{*}$ and $\mathbf{x}^{*}=\mathbf{w}^{*}$, we can further get

$$
\left\|\mathbf{A} \mathbf{c}^{*}-\mathbf{x}^{*}\right\|_{2}^{2} \leq \frac{1}{\tilde{\rho}}\left(\left\|\mathbf{S}_{\mathrm{D}}\left(\mathbf{c}^{1}-\mathbf{c}_{\mathrm{o}}\right)\right\|_{2}^{2}-\left\|\mathbf{S}_{\mathrm{D}}\left(\mathbf{c}^{*}-\mathbf{c}_{\mathrm{o}}\right)\right\|_{2}^{2}\right)=\mathcal{O}\left(\frac{1}{\tilde{\rho}}\right),
$$

which concludes the proof of the third part of Theorem 2

\section{APPENDIX E \\ PROOF OF THEOREM 3}

To be clear, here we rewrite (54)

$$
\begin{aligned}
& L_{\rho}\left(\mathbf{c}^{k}, \mathbf{x}^{k}, \mathbf{u}^{k}, \mathbf{w}^{k}, \mathbf{y}_{1}^{k}, \mathbf{y}_{2}^{k}\right) \\
& \quad-L_{\rho}\left(\mathbf{c}^{k+1}, \mathbf{x}^{k+1}, \mathbf{u}^{k+1}, \mathbf{w}^{k+1}, \mathbf{y}_{1}^{k+1}, \mathbf{y}_{2}^{k+1}\right) \\
\geq & \lambda_{\min }(\mathbf{Q})\left(\left\|\mathbf{u}^{k+1}-\mathbf{u}^{k}\right\|_{2}^{2}+\left\|\mathbf{w}^{k+1}-\mathbf{w}^{k}\right\|_{2}^{2}\right) .
\end{aligned}
$$

Summing both sides of the above inequality from $k=$ $1, \cdots, K$, we have

$$
\begin{aligned}
& \quad L_{\rho}\left(\mathbf{c}^{1}, \mathbf{x}^{1}, \mathbf{u}^{1}, \mathbf{w}^{1}, \mathbf{y}_{1}^{1}, \mathbf{y}_{2}^{1}\right) \\
& \quad-L_{\rho}\left(\mathbf{c}^{K+1}, \mathbf{x}^{K+1}, \mathbf{u}^{K+1}, \mathbf{w}^{K+1}, \mathbf{y}_{1}^{K+1}, \mathbf{y}_{2}^{K+1}\right) \\
& \geq \lambda_{\min }(\mathbf{Q}) \sum_{k=1}^{K}\left(\left\|\mathbf{u}^{k+1}-\mathbf{u}^{k}\right\|_{2}^{2}+\left\|\mathbf{w}^{k+1}-\mathbf{w}^{k}\right\|_{2}^{2}\right) .
\end{aligned}
$$

Since $r=\min \left\{k \mid\left\|\mathbf{u}^{k+1}-\mathbf{u}^{k}\right\|_{2}^{2}+\left\|\mathbf{w}^{k+1}-\mathbf{w}^{k}\right\|_{2}^{2} \leq \epsilon\right\}$, we can change (72) to

$$
\begin{aligned}
& \quad L_{\rho}\left(\mathbf{c}^{1}, \mathbf{x}^{1}, \mathbf{u}^{1}, \mathbf{w}^{1}, \mathbf{y}_{1}^{1}, \mathbf{y}_{2}^{1}\right) \\
& \quad \quad-L_{\rho}\left(\mathbf{c}^{r+1}, \mathbf{x}^{r+1}, \mathbf{u}^{r+1}, \mathbf{w}^{r+1}, \mathbf{y}_{1}^{r+1}, \mathbf{y}_{2}^{r+1}\right) \\
& \geq
\end{aligned}
$$

Since we have $L_{\rho}\left(\mathbf{c}^{r+1}, \mathbf{x}^{r+1}, \mathbf{u}^{r+1}, \mathbf{w}^{r+1}, \mathbf{y}_{1}^{r+1}, \mathbf{y}_{2}^{r+1}\right) \geq$ $L_{\rho}\left(\mathbf{c}^{*}, \mathbf{x}^{*}, \mathbf{u}^{*}, \mathbf{w}^{*}, \mathbf{y}_{1}^{*}, \mathbf{y}_{2}^{*}\right),(73)$ can be reduced to

$$
\begin{aligned}
r \leq \frac{1}{C \epsilon}\left(L_{\rho}\left(\mathbf{c}^{1}, \mathbf{x}^{1}, \mathbf{u}^{1}, \mathbf{w}^{1}, \mathbf{y}_{1}^{1}, \mathbf{y}_{2}^{1}\right)\right. \\
\left.-L_{\rho}\left(\mathbf{c}^{*}, \mathbf{x}^{*}, \mathbf{u}^{*}, \mathbf{w}^{*}, \mathbf{y}_{1}^{*}, \mathbf{y}_{2}^{*}\right)\right),
\end{aligned}
$$

where $C=\lambda_{\min }(\mathbf{Q})$, and $L_{\rho}\left(\mathbf{c}^{*}, \mathbf{x}^{*}, \mathbf{u}^{*}, \mathbf{w}^{*}, \mathbf{y}_{1}^{*}, \mathbf{y}_{2}^{*}\right)=$ $\frac{1}{2}\left\|\mathbf{S}_{\mathrm{D}}\left(\mathbf{c}^{*}-\mathbf{c}_{\mathrm{o}}\right)\right\|_{2}^{2}+\frac{\tilde{\rho}}{2}\left\|\mathbf{u}^{*}-\mathbf{w}^{*}\right\|_{2}^{2}$, which concludes the proof of Theorem 3

\section{REFERENCES}

[1] J. Bingham, "Multicarrier modulation for data transmission: an idea whose time has come", IEEE Commun. Mag., vol. 28, no. 5, pp. 5-14, May. 1990.

[2] S. H. Han and J. H. Lee, "An overview of peak-to-average power ratio reduction techniques for multicarrier transmission", IEEE Wireless Commun., vol. 12, no. 2, pp. 56-65, Apr. 2005.

[3] Y. Rahmatallah and S. Mohan, "Peak-to-average power ratio reduction in OFDM systems: a survey and taxonomy", IEEE Commun. Surveys Tuts., vol. 15, no. 4, Fourth Quarter 2013.

[4] N. Taspinar and M. Yildirim, "A novel parallel artificial bee colony algorithm and its PAPR reduction performance using SLM scheme in OFDM and MIMO-OFDM systems", IEEE Commun. Lett., vol 19, no. 10, pp. 1830-1833, Oct. 2015.

[5] Y. J. Cho, K. H. Kim, and J. Y. Woo, "Low-complexity PTS schemes using dominant time-domain samples in OFDM systems", IEEE Trans. Broadcast., vol. 63, no. 2, pp. 440-445, June. 2017.

[6] T. Jiang, C. Ni, C. Xu, and Q. Qi, "Curve fitting based tone reservation method with low complexity for PAPR reduction in OFDM systems", IEEE Commun. Lett., vol. 18, no. 5, pp. 805-808, May. 2014. 
[7] R. Yoshizawa, and H. Ochiai, "A trellis shaping for peak and average power reduction of BICM-OFDM signals", IEEE Trans. Wireless Commun., vol. 14, no. 2, pp. 1143-1154, Feb. 2015.

[8] S. Shu, D. Qu, L. Li, and T. Jiang, "Invertible subset QC-LDPC codes for PAPR reduction of OFDM signals", IEEE Trans. Broadcast., vol. 61, no. 2, pp. 290-298, June. 2015.

[9] M. Noshad and M. Brandt-Pearce, "Hadamard-coded modulation for visible light communications", IEEE Trans. Commun., vol. 64, no. 3, pp. 1167-1175, Mar. 2016.

[10] J. Armstrong, "Peak-to-average power reduction for OFDM by repeated clipping and frequency domain filtering", Electron. Lett., vol. 38, no. 5, pp. 246-247, Feb. 2002.

[11] M.-X. Hu, Y.-Z. Li, W. Wang, and H.-L. Zhang, "A piecewise linear companding transform for PAPR reduction of OFDM signals with companding distortion mitigation", IEEE Trans. Broadcast., vol. 60, no. 3, pp. 532-539, Sep. 2014.

[12] J. Hou, J.-H. Ge, and F.-K. Gong, "Tone reservation technique based on peak-windowing residual noise for PAPR reduction in OFDM systems", IEEE Trans. Veh. Technol., vol. 64, no. 11, pp. 5373-5378, Nov. 2015.

[13] J.-J. Song and H. Ochiai, "Performance analysis for OFDM signals with peak cancellation", IEEE Trans. Commun. vol. 64, no. 1, pp. 261-270, Jan. 2016.

[14] A. Aggarwal and T. Meng, "Minimizing the peak-to-average power ratio of OFDM signals using convex optimization", IEEE Trans. Signal Process., vol. 54, no. 8, pp. 3099-3110, Aug. 2006.

[15] Y.-C. Wang and Z.-Q. Luo, "Optimized iterative clipping and filtering for PAPR reduction of OFDM signals", IEEE Trans. Commun., vol. 59, no. 1, pp. 33-37, Jan. 2011.

[16] Q.-J. Liu, R. Baxley, X. Ma, and G.-T. Zhou, "Error vector magnitude optimization for OFDM systems with a deterministic peak-to-average power ratio constraint", IEEE J. Sel. Topics Signal Process., vol.3, no.3, pp. 418-429, June. 2009.

[17] X.-D. Zhu, W.-S. Pan, H. Li, and Y.-X. Tang, "Simplified approach to optimized iterative clipping and filtering for PAPR reduction of OFDM signals", IEEE Trans. Commun., vol. 61, no. 5, pp. 1891-1901, May. 2013.

[18] L. Anoh, C. Tanriover, and B. Adebisi, "On the optimization of iterative clipping and filtering for PAPR reduction in OFDM systems", IEEE Access, vlo. 5, pp. 12004-12013, 2017.

[19] Y.-C. Wang, J.-L. Wang, K.-C. Yi, and B. Tian, "PAPR reduction of OFDM signals with minimized EVM via semidefinite relaxation", IEEE Trans. Veh. Technol., vol. 60, no. 9, pp. 4662-4667, Nov. 2011.

[20] S. Boyd, N. Parikh, E. Chu, B. Peleato, and J. Eckstein, "Distributed optimization and statistical learning via the alternating direction method of multipliers", Foundations and Trends in Machine Learning, vol. 3, no. 1, pp. 1-122, 2010

[21] S. Boyd and L. Vandenberghe, "Convex optimization", New York: Cambridge University Press, 2004, pp. 650-652.

[22] M.-Y. Hong and Z.-Q. Luo, "On the linear convergence of the alternating direction method of multiplier", Mar. 2013 [Online]. Available: http://arxiv.org/abs/1208.3922

[23] T. Goldstein, B. O'Donoghue, S. Setzer, and R. Baraniuk, "Fast alternating direction optimization methods", SIAM Journal on Imaging Sciences, vol. 7, no. 3, pp. 225-231, 2014.

[24] J. Sturm, "Using sedumi 1.02, a matlab toolbox for optimization over symmetric cones", Optimiz. Math. Softw., vol. 11-12, pp. 625-653, 1999.

[25] M. Grant and S. Boyd, "CVX: matlab software for disciplined convex programming (Web page and software)", Oct. 2008 [Online]. Available: http://stanford.edu/-boyd/cvx

[26] Y. Wang, W. Yin, and J. Zeng, "Global convergence of ADMM in nonconvex nonsmooth optimization", [Online]. Available: https://arxiv.org/abs/1511.06324.

[27] M.-Y. Hong, Z.-Q. Luo, and M. Razaviyayn, "Convergence analysis of alternating direction method of multipliers for a family of nonconvex problem" [Online], Available: http://arxiv.org/abs/1410.1390, Nov. 2015.

[28] V. Sudha, B. Anilkumar, M. S. Samatha, and D. S. Kumar, "A lowcomplexity modified SLM with new phase sequences for PAPR reduction in OFDM system", in Annual IEEE India Conf., Dec. 2015, pp. 1-5.

[29] W. Wang, M.-X. Hu, Y.-Z. Li, and H.-L. Zhang, "A low-complexity tone injection scheme based on distortion signals for PAPR reduction in OFDM systems", IEEE Trans. Broadcast., vol. 62, no. 4, Dec. 2016.

[30] Y. Sun, D. W. K. Ng, Z. Ding, and R. Schober, "Optimal joint power and subcarrier allocation for full-duplex multicarrier non-orthogonal multiple access systems," IEEE Trans. Commun., vol. 65, no. 3, pp. 1077-1091, Mar. 2017. 